\title{
The occurrence of Listeria monocytogenes is associated with built environment microbiota in three tree fruit processing facilities
}

\author{
Xiaoging Tan ${ }^{1,2}$, Taejung Chung ${ }^{1,2}$, Yi Chen ${ }^{3}$, Dumitru Macarisin ${ }^{3}$, Luke LaBorde ${ }^{1}$ and Jasna Kovac ${ }^{1,2^{*}}$ (D)
}

\begin{abstract}
Background: Multistate foodborne disease outbreaks and recalls of apples and apple products contaminated with Listeria monocytogenes demonstrate the need for improved pathogen control in the apple supply chain. Apple processing facilities have been identified in the past as potential sources of persisting L. monocytogenes contamination. In this study, we sought to understand the composition of microbiota in built apple and other tree fruit processing environments and its association with the occurrence of the foodborne pathogen L. monocytogenes.

Results: Analysis of 117 samples collected from three apple and other tree fruit packing facilities (F1, F2, and F3) showed that facility F2 had a significantly higher L. monocytogenes occurrence compared to F1 and F3 $(p<0.01)$. The microbiota in facility F2 was distinct compared to facilities F1 and F3 as supported by the mean Shannon index for bacterial and fungal alpha diversities that was significantly lower in F2, compared to F1 and F3 $(p<0.01)$. Microbiota in F2 was uniquely predominated by bacterial family Pseudomonadaceae and fungal family Dipodascaceae.

Conclusions: The composition and diversity of microbiota and mycobiota present in the investigated built food processing environments may be indicative of persistent contamination with $L$. monocytogenes. These findings support the need for further investigation of the role of the microbial communities in the persistence of $L$. monocytogenes to support the optimization of L. monocytogenes control strategies in the apple supply chain.
\end{abstract}

Keywords: Listeria monocytogenes, Microbiota, Mycobiota, Fruit, Built food processing environment

\section{Background}

Listeriosis is a foodborne infectious disease caused by Listeria monocytogenes. Listeriosis has $20 \%$ to $30 \%$ fatality rate in high-risk groups, such as elderly, pregnant woman, and immunocompromised individuals [1]. L. monocytogenes infections have been historically associated with outbreaks traced back to ready-to-eat meat products [2] and unpasteurized raw milk [3]. However, in recent years, an increased number of listeriosis outbreaks have been linked with contaminated fresh produce $[4,5]$. In 2014, a multistate outbreak of listeriosis traced back to contaminated

\footnotetext{
* Correspondence: jzk303@psu.edu

${ }^{1}$ Department of Food Science, The Pennsylvania State University, University Park, PA 16802, USA

${ }^{2}$ Microbiome Center, Huck Institutes of the Life Sciences, The Pennsylvania

State University, University Park, PA 16802, USA

Full list of author information is available at the end of the article
}

prepackaged caramel apples caused 34 hospitalizations and seven deaths [6]. In 2017, three more cases of listeriosis associated with prepackaged caramel apples were reported [7]. Several recalls of sliced apples contaminated with $L$. monocytogenes have raised broader concerns about the safety of apples, primarily when further processing provides suitable conditions for $L$. monocytogenes growth $[8,9]$. Increased food safety scrutiny has led to enhanced monitoring of L. monocytogenes in tree fruit packing and processing facilities, and there is a need to investigate factors that may play a role in the establishment and persistence of $L$. monocytogenes in built environments in the apple supply continuum.

A number of studies have investigated the effects of commonly used sanitizers on the reduction of $L$. monocytogenes and on its ability to adapt to these 
antimicrobial treatments [10-13]. These studies provided valuable insight into the ability of sanitizers to inhibit the growth of L. monocytogenes in monocultures $[10,14]$; however, they failed to adequately model the complex biotic environmental conditions found in postharvest food processing built environments $[15,16]$. It is becoming increasingly evident that microbiota found in food production and processing environments plays a role in pathogen survival and persistence and that microbiota needs to be taken in consideration when assessing the effectiveness of pathogen control strategies [16].

Interspecies interactions between $L$. monocytogenes and other microorganisms that make up the food processing environment microbiota have been shown to alter the ability of L. monocytogenes to survive and colonize facilities [17]. For example, Pseudomonas spp. commonly found in food processing environments [14, 15] has been found to have a positive effect on $L$. monocytogenes attachment on the stainless steel surfaces [18]. In contrast, Staphylococcus sciuri was shown to decrease L. monocytogenes biofilm formation on stainless steel surfaces [19]. Carpentier and Chassaing [20] demonstrated that among 29 bacteria isolated from the dairy and meat processing environment, four strains promoted $L$. monocytogenes growth in the resulting biofilm when co-cultured with L. monocytogenes, whereas 16 strains suppressed the growth of L. monocytogenes. Although the exact mechanisms underlying these biological interactions and phenotypic outcomes are yet to be elucidated, competition for nutrients and production of anti-listerial secondary metabolites have been proposed as important microbiota-shaping factors $[19,21]$. One of the important steps toward gaining a better understanding of microbial interactions in microbial communities is the characterization of environmental microbiota in food production and processing facilities and investigation of associations and co-occurrence of L. monocytogenes and other members of the microbiota.

Amplicon sequencing has revolutionized the characterization of microbial communities, not only in human medicine and ecology but also in the food industry where information about microbiota dynamics can enhance our ability to answer applied questions related to food safety and quality. Microorganisms such as P. psychrophila, Pseudomonas sp., Klebsiella sp., K. oxytoca, and Aeromonas hydrophila have been identified through $16 \mathrm{~S}$ rRNA sequencing as the dominant species in L. monocytogenes-positive drains located in dairy, meat, peanut butter, and spice processing plants, suggesting that they might facilitate $L$. monocytogenes biofilm formation through interspecies interactions [22]. Moreover, studies have been carried out in dairy and meat processing plants, where associations have been found between the indoor bacterial communities and the presence of L. monocytogenes [23, 24]. Food processing facilities that serve as an intermediate between the pre-harvest, raw ingredient, and retail distribution chain have been identified among the main potential sources of pathogen contamination [25-28]. It is therefore critical to establish a baseline understanding of microbial diversity in food processing environments and to investigate the associations between microbial community composition and occurrence of $L$. monocytogenes in these environments. This knowledge can be used to develop targeted microbiota manipulation strategies for the development of improved foodborne pathogen control strategies.

There is only limited data available on the composition of microbial communities in built produce processing environments [29]. To the best of our knowledge, no published studies have reported the relationship between apple and other tree fruit packing house microbiota composition and the presence of $L$. monocytogenes in these environments to date. We, therefore, utilized 16S rRNA V4 and internal transcribed spacer 2 (ITS2) amplicon sequencing coupled with $L$. monocytogenes enrichment to elucidate the associations between the composition, diversity, and predicted functional profiles of the built environment microbiota in three apple and other tree fruit packing facilities to provide new knowledge enabling improvement of food safety.

\section{Methods}

\section{Study design}

Occurrence of Listeria monocytogenes and characterization of microbiota within three apple and other tree fruit packing houses in the Northeast U.S. was monitored through the fruit harvesting and packing season. At each facility, nonfood-contact (zone 3) environmental samples were collected from the floor under a conveyor system with rolling brushes that transported fruit through successive washing, drying, and waxing processes, as outlined below. These specific locations were selected for sampling because preliminary data from season 2016/17 showed a higher occurrence of $L$. monocytogenes in these locations compared to other locations in the packing houses. Based on the preliminary data, we established that a sample size of 117 is needed to identify the prevalence of $L$. monocytogenes with a $7.25 \%$ precision and $95 \%$ confidence [30]. Therefore, a total of 117 samples, 39 samples from each of the three facilities, were collected twice a month over 13 sampling time points between November 2017 and April 2018. Information including the facility code, sampling date, and sampling location within the facility are provided in the Additional file 1: Table S1.

\section{Sample collection}

Non-food contact surface environmental areas $(40 \mathrm{~cm}$ by $40 \mathrm{~cm}$ ) were sampled by swabbing with pre-moistened sponges (3M) with a combination of ten horizontal and ten vertical strokes. Samples for Listeria spp. isolation and identification were collected with $3 \mathrm{M}$ hydrated sponges 
pre-moistened with $10 \mathrm{ml} \mathrm{D} / \mathrm{E}$ neutralizing buffer (3M, cat. number HS10DE2G) to enhance the survival of Listeria through the neutralization of a broader range of sanitizers [31]. The samples for microbiota characterization were collected using $3 \mathrm{M}$ hydrated sponges with just $10 \mathrm{ml}$ of neutralizing buffer (3M, cat. number HS2410NB2G). Two adjacent duplicate areas were sampled at each of the three areas (washing, drying, waxing) under the conveyor belt (Fig. 1). One of the duplicate samples was used for L. monocytogenes enrichment, isolation, and identification, and the other for microbiota and mycobiota characterization using $16 \mathrm{~S}$ rRNA V4 and ITS2 Illumina sequencing. A total of 18 samples were collected per time point; six in each of the three facilities. All swab samples were stored in a cooler on the ice during transportation to the laboratory and were processed on the same day.

\section{Listeria monocytogenes enrichment and isolation}

Detection of Listeria spp. was conducted following a modified Food and Drug Administration bacteriological analytical manual (FDA BAM) protocol for the detection and enumeration of L. monocytogenes [31]. Briefly, $90 \mathrm{ml}$ of buffered Listeria enrichment broth (BLEB, Oxoid) were added to each test sample and control sample, followed by manual homogenization by hand-massaging the sponge for 15 s. L. monocytogenes strain F2365 [32] and Listeria innocua strain PS00298 were used as positive and negative controls in every sample processing batch, respectively, to compare their morphology with the morphology of putative Listeria spp. isolated on selective agars as well as with the resulting polymerase chain reaction (PCR) product separated using gel electrophoresis. A sterile sealed sampling sponge from the same production batch served as an additional negative control to ensure that the used batch of sampling sponges was not contaminated with L. monocytogenes. For the enrichment, $400 \mu \mathrm{l}$ of BLEB supplement SR0149 (Oxoid) were added to each test sample and controls after $4 \mathrm{~h}$ of pre-enrichment in BLEB at $30{ }^{\circ} \mathrm{C}$. After adding the supplement, samples were further incubated for $44 \pm 2 \mathrm{~h}$ at $30{ }^{\circ} \mathrm{C}$. Upon completed incubation, a loopful of each enrichment was streaked onto Agar Listeria Ottavani \& Agosti (ALOA, BioRad Laboratories Inc.) and RAPID' L. mono (BioRad Laboratories Inc.) selective differential agars, and incubated for $48 \mathrm{~h}$ at $37{ }^{\circ} \mathrm{C}$. After incubation, presumptive positive L. monocytogenes colonies appeared blue/green with an opaque white halo on the ALOA agar, whereas presumptive positive L. monocytogenes colonies appeared blue with no background change on RAPID' L. mono agar. Other Listeria spp. may also grow on these selective differential agars; however, only one presumptive L. monocytogenes colony was collected from each, ALOA and RAPID L' mono agars, and streaked for isolation on trypticase soy agar with yeast extract (TSAYE) in this study. Streaked plates were incubated for $24 \mathrm{~h}$ at $37{ }^{\circ} \mathrm{C}$ and isolated colonies were used for PCR-based L. monocytogenes confirmation.

\section{Listeria monocytogenes identification and cryopreservation}

One isolated colony per TSAYE plate was selected, inoculated in the trypticase soy broth with yeast extract (TSBYE), and incubated at $30{ }^{\circ} \mathrm{C}$ for $24 \mathrm{~h}$. One milliliter of each overnight culture was centrifuged at $15,000 \times g$ for $15 \mathrm{~min}$, washed with DNase free water, and centrifuged again at 15 , $000 \times g$ for $15 \mathrm{~min}$. The cell pellets were resuspended in 50 $\mu \mathrm{l}$ of DNase free water and heated for $10 \mathrm{~min}$ at $95{ }^{\circ} \mathrm{C}$ to lyse the cells. Lysates were then centrifuged at $15,000 \times g$ for $10 \mathrm{~min}$ to remove the cell debris. Supernatants containing deoxyribonucleic acid (DNA) template were stored at - 20 ${ }^{\circ} \mathrm{C}$ until further use. Two microliters of DNA template were used in a PCR reaction with primers targeting genes iap (specific for Listeria spp.) and lmo2234 (specific for $L$. monocytogenes) to confirm the L. monocytogenes species [32]. L. monocytogenes strain F2365 and Listeria innocua strain PS00298 served as positive controls and the nuclease-free water was used as a negative control. The

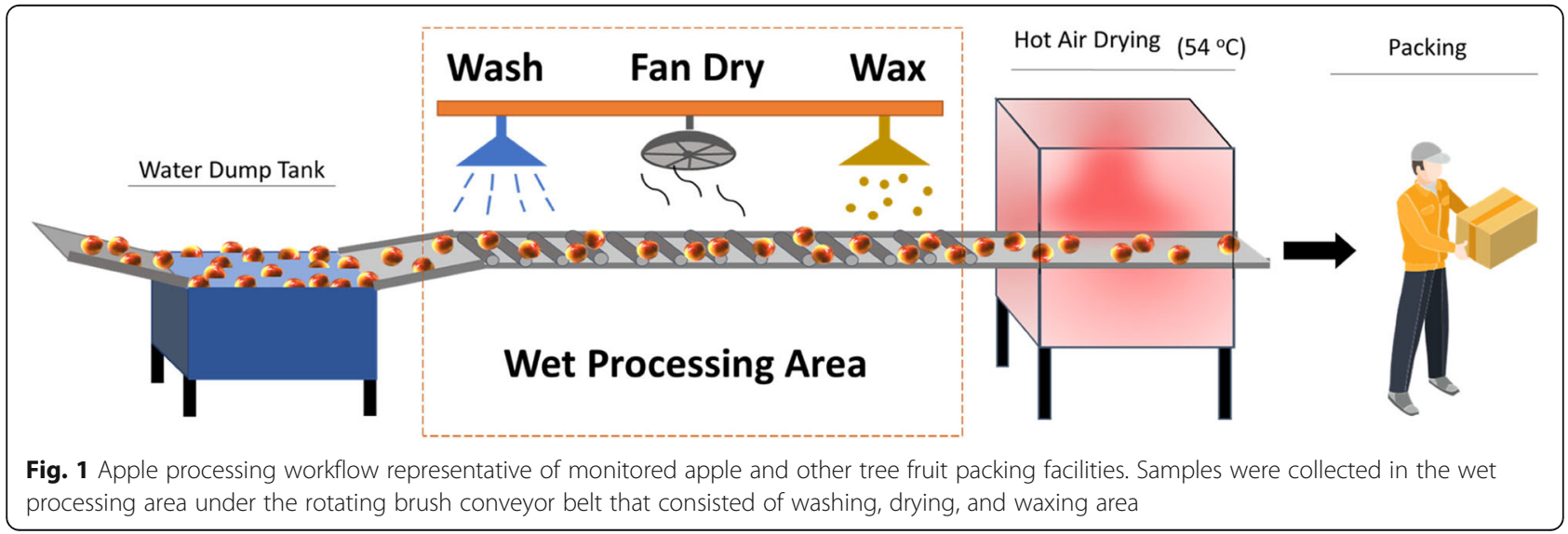


following thermal cycling conditions were used: initial denaturation at $95{ }^{\circ} \mathrm{C}$ for $15 \mathrm{~min}, 15$ cycles of denaturation at $94{ }^{\circ} \mathrm{C}$ for $1 \mathrm{~min}$, annealing at $55{ }^{\circ} \mathrm{C}$ to $51{ }^{\circ} \mathrm{C}$ for $1 \mathrm{~min}$ with a touchdown of 3 cycles per temperature, extension at 72 ${ }^{\circ} \mathrm{C}$ for $1 \mathrm{~min}$. The following 15 cycles started with denaturation at $94{ }^{\circ} \mathrm{C}$ for $1 \mathrm{~min}$, annealing at $50{ }^{\circ} \mathrm{C}$ for $1 \mathrm{~min}$, extension at $72{ }^{\circ} \mathrm{C}$ for $1 \mathrm{~min}$, and the final extension at $72{ }^{\circ} \mathrm{C}$ for 8 min [32]. Successful PCR amplification was confirmed by gel electrophoresis using $2 \%$ agarose gel (Invitrogen). A band between 1450 and 1600 bp (for iap) and a band at 420 bp (specific for lmo2234) were expected in samples positive for Listeria spp. or L. monocytogenes, respectively. Isolates that were confirmed as Listeria spp. or $L$. monocytogenes were re-streaked on TSAYE and grown overnight in TSBYE. Overnight cultures were supplemented with $20 \%$ glycerol and stored in $-80{ }^{\circ} \mathrm{C}$ freezer.

\section{Quantification of Listeria using the most probable number assay}

Most probable number (MPN) assay for L. monocytogenes was carried out on 18 samples collected in two samplings conducted in March 2018 to determine the level of $L$. monocytogenes contamination, using the enrichment media described above. Briefly, $90 \mathrm{ml}$ of BLEB were added to a bag containing a sponge sample, and manually homogenized. One milliliter of the homogenate was added directly to $9 \mathrm{ml}$ of BLEB, in triplicate, and enriched. Another $1 \mathrm{ml}$ was serially tenfold diluted four times in $9 \mathrm{ml}$ of PBS, in triplicates. One milliliter of each of these four serial dilutions were transferred into test tubes containing $9 \mathrm{ml}$ of BLEB and enriched. This resulted in five MPN dilutions ranging from $10^{-2}$ (1/100 of homogenate) to $10^{-6}$ of the original sample. The remaining homogenate was enriched as well. Forty microliters of BLEB supplement were added to each tube after $4 \mathrm{~h}$ of incubation at $30{ }^{\circ} \mathrm{C}$. Supplemented samples were further incubated for $44 \pm 2$ $\mathrm{h}$ at the same temperature. After 48-h incubation, a loopful of each dilution enrichment was streaked onto ALOA and RAPID' L. mono agar plates and incubated at $37{ }^{\circ} \mathrm{C}$ for 24 to $48 \mathrm{~h}$, until obtaining visible colonies. The MPN of $L$. monocytogenes per sponge sample was determined using an MPN calculator provided in the Excel file available for download in "BAM: Detection and Enumeration of Listeria monocytogenes" [33].

\section{Total DNA extraction for microbiota and mycobiota analysis}

Each environmental sponge sample collected for microbiota characterization was homogenized with $50 \mathrm{ml}$ of phosphate buffer containing $0.9 \% \mathrm{NaCl}$ in a stomacher, for $7 \mathrm{~min}$ at $230 \mathrm{rpm}$. Fifty milliliters of the homogenate were transferred to a sterile $50 \mathrm{ml}$ conical tube and centrifuged at $11,000 \times g$ and $4{ }^{\circ} \mathrm{C}$ for 20 min (Beckman Coulter, Avanti J-26 XPI) [34]. After centrifugation, supernatants were discarded and pellets were stored at $-80{ }^{\circ} \mathrm{C}$ until DNA extraction. DNA was extracted from approximately $0.25 \mathrm{~g}$ of each sample using DNeasy PowerSoil DNA extraction kit (QIAGEN) following manufacturer's protocol. Approximately $0.25 \mathrm{~g}$ of a sterile sponge was also sampled and used as a negative control to confirm the absence of microbial DNA contaminants on the sterile sponge. DNA extracted from the sponge was processed following the same protocol as described below for other samples. The concentration of DNA in each test sample and in the control sample was determined both spectrophotometrically using NanoDrop One (Thermo Scientific) and fluorometrically using Qubit 3 (Invitrogen) and Qubit dsDNA High Sensitivity Assay Kit. DNA samples were stored at $-80{ }^{\circ} \mathrm{C}$ until further use.

\section{S rRNA V4 and ITS2 sequence amplification}

Bacterial and fungal community composition was determined by targeted metagenomic sequencing of the PCRamplified V4 domain of the 16S rRNA gene and the internal transcribed spacer 2 (ITS2) sequences, respectively. Briefly, V4 region of the 16S rRNA gene sequence was amplified using KAPA HiFi HotStart ReadyMix (Kapa Biosystems), a forward primer 505F-v2 (TCG TCG GCA GCG TCA GAT GTG TAT AAG AGA CAG GTG YCA GCM GCC GCG GTA A), and a reverse primer 806R-v2 (GTC TCG TGG GCT CGG AGA TGT GTA TAA GAG ACA GGG ACT ACN VGG GTW TCT AAT) [35]. PCR thermal cycling for amplification of the 16S rRNA gene V4 region was conducted as follows: initial denaturation at $95{ }^{\circ} \mathrm{C}$ for $3 \mathrm{~min}, 29$ cycles of denaturation at $98^{\circ} \mathrm{C}$ for $30 \mathrm{~s}$, annealing at $55^{\circ} \mathrm{C}$ for $30 \mathrm{~s}$, extension at $72{ }^{\circ} \mathrm{C}$ for 30 $\mathrm{s}$, and the final extension at $72{ }^{\circ} \mathrm{C}$ for $5 \mathrm{~min}$, and final hold at $4{ }^{\circ} \mathrm{C}$. Fungal internal transcribed spacer (ITS) loci were amplified using a forward primer ITS4F (TCG TCG GCA GCG TCA GAT GTG TAT AAG AGA CAG GAA CGC AGC RAA IIG YGA) and a reverse primer ITS9R (GTC TCG TGG GCT CGG AGA TGT GTA TAA GAG ACA GTC CTC CGC TTA TTG ATA TGC) [36]. PCR conditions for ITS amplification were as follows: initial denaturation at $98^{\circ} \mathrm{C}$ for $5 \mathrm{~min}, 30$ cycles of denaturation at $95^{\circ} \mathrm{C}$ for $45 \mathrm{~s}$, annealing at $50{ }^{\circ} \mathrm{C}$ for $60 \mathrm{~s}$, extension at $72{ }^{\circ} \mathrm{C}$ for $60 \mathrm{~s}$, and the final extension at $72{ }^{\circ} \mathrm{C}$ for $5 \mathrm{~min}$, and the final hold at $4{ }^{\circ} \mathrm{C}$. PCR amplicons were visualized by running gel electrophoresis using a $2 \%$ agarose gel to confirm successful amplification of target sequences.

\section{Amplicon library preparation and amplicon sequencing}

Amplicon libraries were prepared based on Illumina's 16S Metagenomic Sequencing Library Preparation protocol [37]. 16S rRNA V4 and ITS2 PCR amplicons were barcoded with unique combinations of i7 and i5 index adaptors (Integrated DNA Technologies) in a 
second-step PCR using following thermal cycling conditions: initial denaturation at $95{ }^{\circ} \mathrm{C}$ for $3 \mathrm{~min}, 8$ cycles of denaturation at $95{ }^{\circ} \mathrm{C}$ for $30 \mathrm{~s}$, annealing at $55^{\circ} \mathrm{C}$ for 30 $\mathrm{s}$, extension at $72{ }^{\circ} \mathrm{C}$ for $30 \mathrm{~s}$, and final extension at 72 ${ }^{\circ} \mathrm{C}$ for $5 \mathrm{~min}$; final hold was at $4{ }^{\circ} \mathrm{C}$. Barcoded PCR amplicon libraries were purified twice with AmPure XP beads (Beckman Coulter) and then normalized using Mag-Bind EquiPure Library Normalization Kit (Omega Bio-tek) following manufacturer's protocol. Concentrations of a subset of normalized libraries were verified using a high sensitivity double-stranded DNA kit with Qubit 3. Libraries were then pooled in equal volumes of $4 \mu \mathrm{l}$. The distribution of pooled library fragment sizes was verified using Bioanalyzer and its concentration using qPCR. Estimated amplicon lengths of 359 bp and $425 \mathrm{bp}$ were used in the calculation of molar concentration and normalization. The library pool was denatured by diluting $5 \mu \mathrm{l}$ of $4 \mathrm{nM}$ library pool with $5 \mu$ l freshly prepared $0.2 \mathrm{~N} \mathrm{NaOH}$. The denatured library pool was diluted with a pre-chilled HT1 buffer to $7.5 \mathrm{pM}$ with $10 \%$ PhiX internal control library. A total of $600 \mu \mathrm{l}$ of denatured library pool spiked with PhiX was loaded onto the Illumina Miseq flow cell. Five hundred cycle V2 Illumina sequencing kit was used for 250 bp paired-end sequencing in two Illumina MiSeq sequencing runs. Sequencing reads were deposited in NCBI SRA under BioProject PRJNA527988.

\section{Sequence analyses and OTU normalization}

Sequences were analyzed with Mothur v1.39.5 following protocols described in Schloss et al. [38, 39] with default settings unless otherwise noted. The workflow of analyses is described in the Additional file 1: List L1. Paired end sequence reads were assembled into contigs. Contigs shorter or longer than $292 \mathrm{bp}$ for $16 \mathrm{~S}$ rRNA V4 bacterial sequences and longer than $350 \mathrm{bp}$ for ITS2 fungal sequences were discarded. The remaining reads were aligned against SILVA database [40] for $16 \mathrm{~S}$ rRNA V4 region of bacterial sequences, and UNITE database [41] for ITS2 fungal sequences. Chimera were detected and discarded using UCHIME algorithm. Operational taxonomic units (OTUs) were calculated using opticlust with $97 \%$ similarity threshold. All 16S rRNA sequences were rarefied to 4501 and ITS sequences were rarefied to 5232 randomly sampled OTUs. Rarefied sequence cutoffs were chosen based on the lowest reads obtained for microbiota and mycobiota samples, in order to include all samples in downstream comparative analyses.

\section{Diversity of microbiota and mycobiota}

Alpha and beta diversity indices were calculated based on rarefied OTUs. For alpha diversity, Shannon and Inverse Simpson indices were calculated for both bacterial and fungal communities. For beta diversity, the weighted
UniFrac distance between either bacterial or fungal communities was calculated in $\mathrm{R}$ using package Phyloseq [42]. Principal coordinates analysis ( $\mathrm{PCoA}$ ) was used to visualize the beta diversity of microbiota with reduced dimensionality. Principal coordinates were plotted using $\mathrm{R}$ package ggplot2 [43]. Chi-square and Fisher's exact tests with Bonferroni correction were used to test the significance of differences in L. monocytogenes occurrence among tree frui packing facilities. Pairwise permutational multivariate analysis of variance (PERMANOVA) test was carried out using package PairwiseAdonis [44] and BrayCurtis dissimilarity matrices were used to test the significance of differences in microbiota and mycobiota composition. Pairwise comparisons were carried out by sampling month, processing section, and facility.

\section{The co-occurrence of microbial and fungal taxa}

Network analyses were carried out to identify significantly co-occurring bacterial families among samples collected from three apple and other tree fruit packing facilities. Networks were constructed using Cytoscape v3.7.1 [45] with CoNet version 1.1.1 beta plug-in [46]. Rarefied 16S rRNA OTU table was condensed to family level taxa and used as an input matrix. Network analysis was conducted based on microbial family occurrence for each individual facility as well as for all three facilities combined. Co-occurrence of specific families was determined using Pearson and Spearman correlations, mutual information, and Bray-Curtis, and Kullback-Leibler dissimilarity indices. Row-shuffle randomization and bootstrap method were used to minimize composition-induced false correlations. The resulting $p$ values were merged using Brown's method and corrected by Benjamini-Hochberg multiple comparison corrections. Interactions were visualized using yfiles layout algorithm plug-in application [47].

\section{Prediction of microbiota's functional profiles}

Raw 16S rRNA sequence reads were re-analyzed using Mothur pipeline outlined above, only this time in conjunction with Greengenes 16S rRNA database version13.8 [48] since Phylogenetic Investigation of Communities by Reconstruction of Unobserved States (PICRUSt) [49] required the use of this database. The biom file resulting from Mothur analysis, and containing OTU and taxonomy information was used as an input file for PICRUSt analysis using online Galaxy-based version provided by Hutlab [50]. Briefly, OTU table was first corrected for $16 \mathrm{~S}$ rRNA copy number by dividing OTU counts by the number of 16S rRNA marker gene copy to obtain an abundance estimation for each OTU. Each Kyoto Encyclopedia of Gene and Genomes (KEGG) [51] ortholog (KO) was then multiplied by the normalized OTU abundance for metagenome prediction. $\mathrm{KO}$ tier 3 was assigned for functional prediction and tier 1 assignments were used for the 
categorization of functional profiles for each facility. The output data table contained summed predicted $\mathrm{KO}$ functional gene abundance per metagenome sample.

\section{Results}

Samples collected from facility F2 had higher occurrence and level of L. monocytogenes compared to those collected from F1 and F3

A total of 117 environmental samples were tested for the presence of L. monocytogenes using the FDA BAM enrichment protocol. Out of 39 samples collected in each facility, L. monocytogenes culture was isolated and confirmed in 11, 39, and 16 samples from facilities. F1, F2, and F3, respectively (Fig. 2). All samples in facility F2 were positive for $L$. monocytogenes using the culturing method, while $11(28 \%)$ and $16(41 \%)$ samples were positive in facilities F1 and F3, respectively. The difference in occurrence of $L$. monocytogenes among facilities was statistically significant $(p<0.001)$. However, there was no significant difference in L. monocytogenes occurrence when comparing washing, fan-drying, and waxing sections $(p=0.112)$. Results of statistical analyses are provided in Additional file 1: Tables S2 and S3.

Quantification of L. monocytogenes in a subset of samples collected on 2 days in March 2018 indicated highlevel contamination of F2 at both sampling times. Samples collected underneath the conveyor belt in washing, fandrying, and waxing sections of the facility F2 contained an average of 3.51, 4.18, and $4.87 \log _{10} \mathrm{MPN} /$ sponge, while an average of $1.50,0.82$, and $2.00 \log _{10} \mathrm{MPN} /$ sponge were found in respective areas in facility F3. No L. monocytogenes was detected in samples collected from facility F1 in the two sampling time points (Table 1). Samples collected from three facilities had significantly different levels of $L$. monocytogenes $(p<0.001)$, with samples collected from F2 having higher-level of contamination compared to those collected from F1 and F3.

$16 \mathrm{~S}$ rRNA and ITS2 were rarefied to the lowest OTU count prior to downstream analyses

In the first sequencing run, a total of $8,298,24016 \mathrm{~S}$ rRNA V4 reads and 8,688,706 ITS2 reads were produced. Samples that were sequenced with fewer than 5000 reads were re-sequenced in a second sequencing run. In the second sequencing run, 7,458,554 $16 \mathrm{~S}$ rRNA reads and 3,485,400 ITS2 reads were produced. For16S rRNA sequences, a minimum, median, and maximum number of total OTUs identified was 4501, 28,056, and 168,219 , respectively, and for ITS2 sequences, a minimum, median, and maximum numbers of total OTUs identified were 5323, 17,882, and 350,415.

Rarefaction curves were plotted using the number of OTUs which represented the sequence sample size, against the number of unique OTUs which represented species richness (Fig. 3). All three facilities generally had a similar sequence sample size; however, richness in samples from facility F2 16S rRNA reached saturation at fewer reads compared to facilities F1 and F3, indicating lower microbial diversity in F2. For ITS2 OTUs, samples from facility F2 had a substantially higher numbers of assembled OTUs compared to samples from F1 and F3. The rarefaction curves for samples from facilities F1 and F3 did not reach saturation, indicating that considerable microbial diversity of low-abundant taxa has not been discovered at a given sequencing depth. Based on the rarefaction curves and our interest in high-abundant

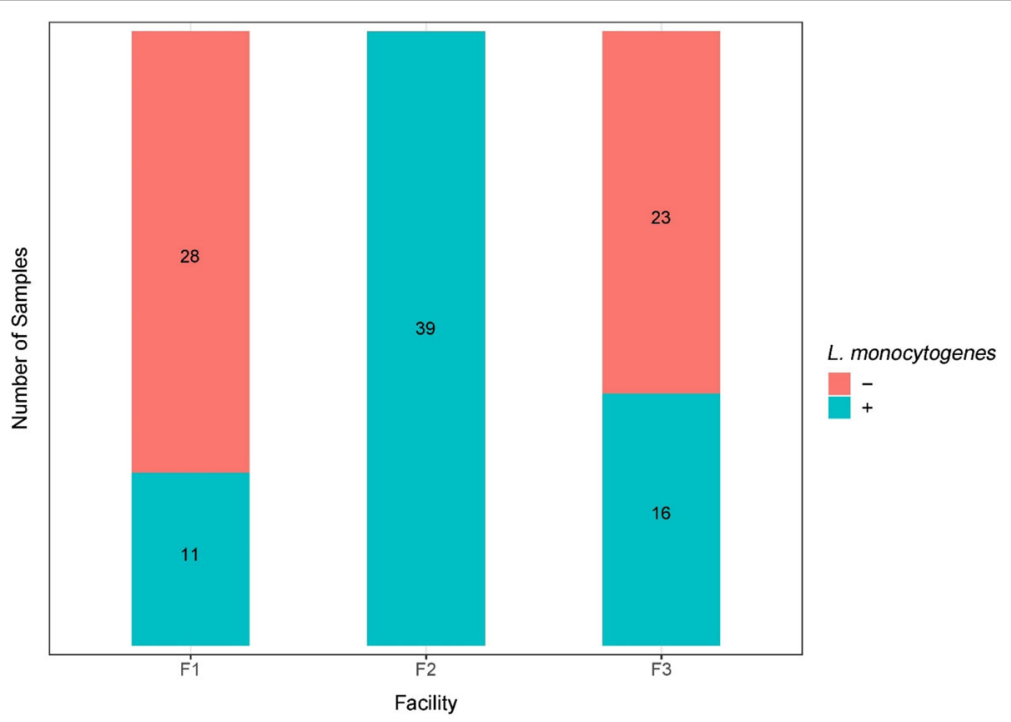

Fig. 2 Summary of the presence $(+)$ and absence (-) of Listeria monocytogenes in environmental samples collected from three tree fruit packing facilities F1, F2, and F3 over the sampling period (November 2017-April 2018), as determined using an enrichment protocol 
Table 1 MPN-based quantification of L. monocytogenes in samples collected from three tree fruit packing facilities at two time points in March 2018

\begin{tabular}{|c|c|c|c|c|c|c|c|c|c|}
\hline \multirow{2}{*}{$\begin{array}{l}\text { Log } \\
\text { MPN/ } \\
\text { sponge } \\
\text { sample }\end{array}$} & \multicolumn{3}{|c|}{ Facility $F 1^{c}$} & \multicolumn{3}{|c|}{ Facility F2 } & \multicolumn{3}{|l|}{ Facility F3 ${ }^{c}$} \\
\hline & Wash & Dry & Wax & Wash & Dry & Wax & Wash & Dry & Wax \\
\hline Trial $1^{\text {a }}$ & $<\mathrm{LD}$ & $<\mathrm{LD}$ & $<L D$ & 3.87 & 5.02 & 4.38 & $\mathrm{LD}<1.55^{\mathrm{d}}$ & $<L D$ & $\operatorname{LD}<1.55^{\mathrm{d}}$ \\
\hline Trial $2^{\mathrm{b}}$ & $<\mathrm{LD}$ & $<L D$ & $<L D$ & 3.15 & 3.32 & 5.35 & 1.55 & 1.55 & 2.63 \\
\hline Average & $<\mathrm{LD}$ & $<L D$ & $<L D$ & 3.51 & 4.17 & 4.87 & $<1.55$ & 0.82 & $<2.63$ \\
\hline
\end{tabular}

${ }^{a}$ Four dilutions $\left(10^{-2}, 10^{-3}, 10^{-4}, 10^{-5}\right)$ were used in the MPN calculation. Each dilution was tested in triplicates. Sample homogenate (a sponge in $90 \mathrm{ml}$ of BLEB) was also enriched

${ }^{\mathrm{b}}$ Five dilutions $\left(10^{-2}, 10^{-3}, 10^{-4}, 10^{-5}, 10^{-6}\right)$ were used in MPN calculation. Each dilution was tested in triplicates. Sample homogenate (a sponge in $90 \mathrm{ml}$ of BLEB) was also enriched

${ }^{c}<$ LD indicates that $L$. monocytogenes was below the limit of detection, which was at least one viable cell per sample, grown to at least $10^{5}$ cells $/ \mathrm{ml}$ by the time the enrichment was completed

${ }^{\mathrm{d}}$ The level of $L$. monocytogenes was between the limit of detection and $1.55 \log _{10}$ MPN/sponge sample. L. monocytogenes was detected in enriched sponge homogenate; however, none of the MPN dilution tubes were positive

taxa that were hypothesized to play a major role in the microbial ecology of monitored environments, we rarefied samples to the lowest number of total OTUs found among sequenced samples (i.e., $N=4501$ for $16 \mathrm{~S}$ rRNA V4 sequences and $N=5323$ for ITS2 sequences).

\section{Alpha diversity of bacterial communities was significantly different in samples collected from facility F2 compared to those collected from facilities F1 and F3}

Microbial and fungal composition of environmental samples collected from three facilities were characterized using rarefied 16S rRNA V4 and ITS2 amplicon sequencing. Alpha diversity indices were visualized using violin plots (Fig. 4) showing microbiota and mycobiota diversity within each individual facility. Significant differences in bacterial alpha diversity were observed among microbiota of samples collected in facilities F1, F3, and F2.
Pairwise $t$ test comparison showed no significant difference in microbial alpha diversity determined based on Inverse Simpson index (Fig. 4a) between facilities F1 and F3 $(p=1.00)$, while significant difference was observed between diversity of microbiota from facilities F1 and F2 $\left(p=1.7 \times 10^{-5}\right)$, as well as from facilities F3 and F2 $(p=$ $\left.1.5 \times 10^{-4}\right)$. Shannon index showed similar trends when comparing the alpha diversity of samples from F1 with samples from F3 $(p=1.00)$. Alpha diversities of samples from F1 $\left(p=6.0 \times 10^{-10}\right)$ and F3 $\left(p=1.5 \times 10^{-9}\right)$ were significantly different from those from facility F2, according to the Shannon index (Fig. 4b).

Alpha diversity indices for fungal communities were also compared among samples collected in three facilities. Significant differences were identified between samples from facilities F1 and F2 $\left(p=7.1 \times 10^{-6}\right)$ as well as samples from F2 and F3 $\left(p=1.6 \times 10^{-4}\right)$ as indicated
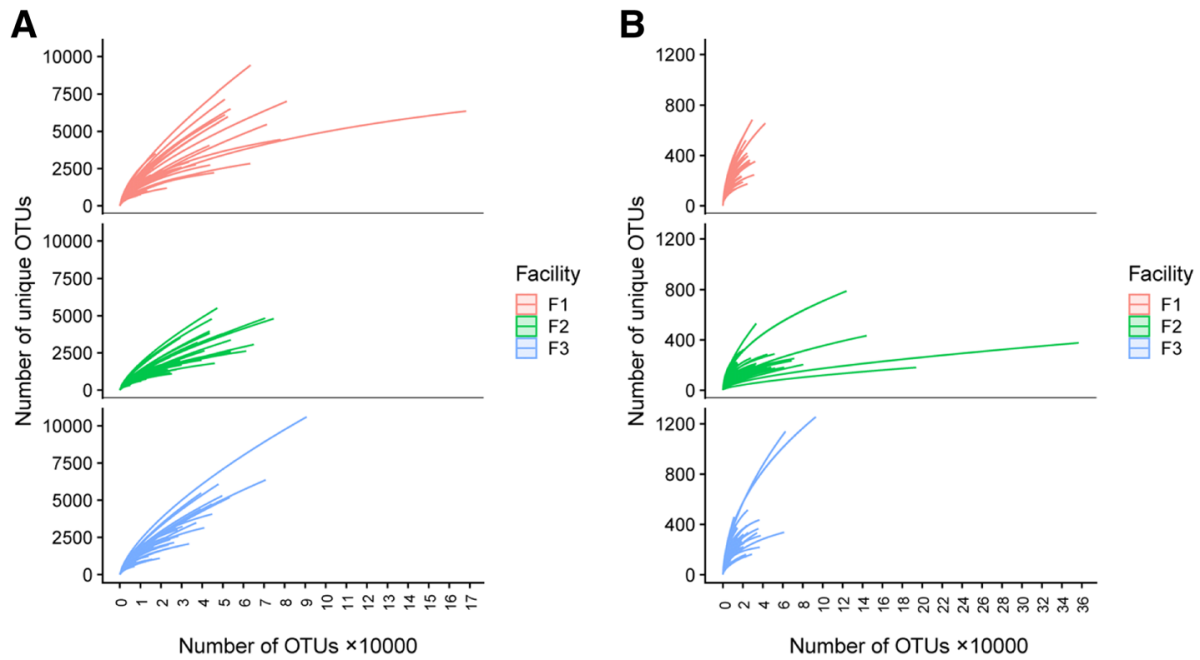

Fig. 3 Rarefaction curves for 16S rRNA V4 (a) and ITS2 (b) sequences. Individual samples were plotted using the number of OTUs that represent the sequence sample size on $x$ axis and the number of unique OTUs that indicate the species richness on $y$ axis. Each curve is color-coded based on the facility in which the sample was collected 
A
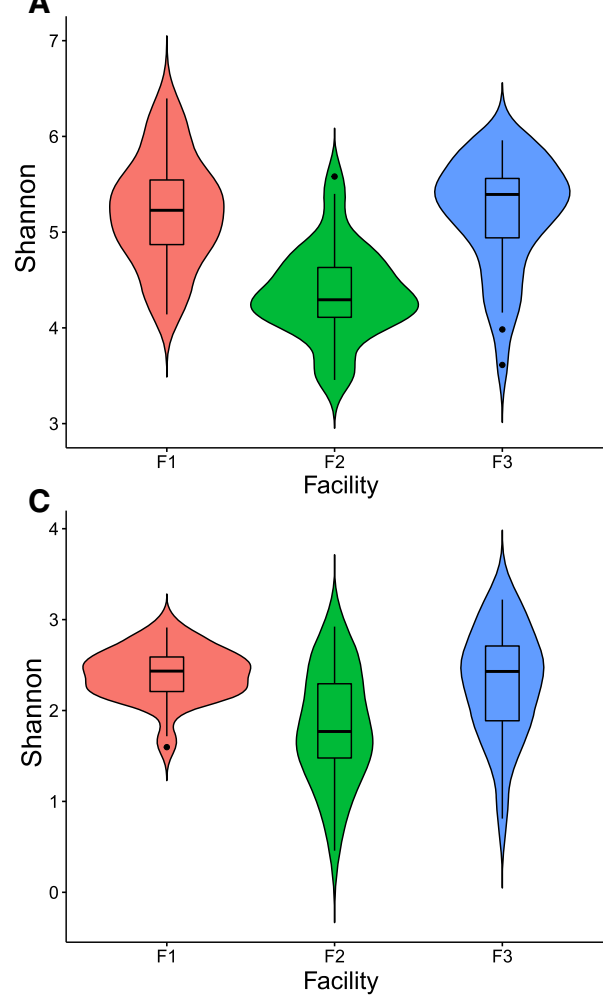

B
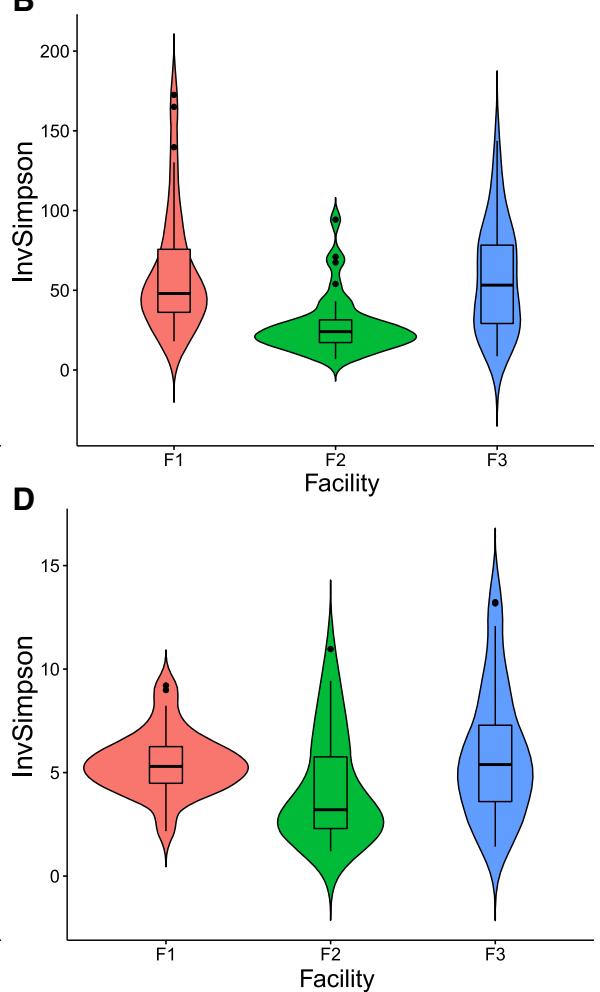

Fig. 4 Microbial alpha diversity distributions determined based on rarefied 16S rRNA V4 and ITS2 assembled contigs for samples collected from three packing facilities, F1, F2 and F3. Alpha diversity was measured using Shannon index (a) and Inverse Simpson index (b). Fungal alpha diversity was determined based on rarefied ITS2 assembled contigs, as measured using Shannon index (c) and Inverse Simpson index (d)

by the Shannon index. No significant difference was observed between the alpha diversity of samples from F1 and F3 ( $p=1.0$, with Bonferroni correction, Fig. 4d). Inverse Simpson index, on the other hand, showed no significant difference between samples from F1 and F2 $(p=0.082)$ nor F1 and F3 $(p=1.00)$. A significant difference, however, was observed between F2 and F3 ( $p=$ 0.011) (Fig. 4c). Overall, the microbial diversity in facility F2 was substantially lower compared to that in facilities $\mathrm{F} 1$ and F3.

\section{Environmental microbiota in facility F2 significantly differed from environmental microbiota in facilities F1 and $\mathrm{F} 3$}

The beta diversity of bacterial communities among facilities was shown in the PCoA plot (Fig. 5). Clustering was analyzed using weighted UniFrac metric which incorporates phylogenetic relatedness when calculating distance among $16 \mathrm{~S}$ rRNA or ITS2 gene sequences. Further, $10.7 \%$ and $6.6 \%$ variances in bacterial composition were represented by the $\mathrm{PC} 1$ and $\mathrm{PC} 2$, respectively. Moreover, $43.1 \%$ and $20.5 \%$ variability in the beta diversity were explained by the PC1 and PC2 for mycobiota. Microbiota of samples collected from facility F2 formed a distinct cluster while microbiota of samples collected from facilities F1 and F3 appeared to be more related to each other than to microbiota from F2 (Fig. 5a). Consistently with distinct F2 microbiota clustering, mycobiota from this facility also appeared to be different compared to that discovered in facilities F1 and F3 (Fig. 5b). However, unlike microbiota, the mycobiota found in samples from facilities F1 and F3 appeared to be less similar (Fig. 5b). In order to further explore the microbial composition that contributed to the distinct clustering of microbiota in the PCoA plot, we investigated the taxonomic composition of collected samples in three individual facilities using PERMANOVA statistical analyses.

\section{Pairwise PERMANOVA analysis indicated that significant differences in microbiota composition exist among facilities}

Beta diversity plot indicated distinct clustering of microbiota among facilities. To statistically evaluate these potential differences, we compared microbiota compositions by the month of sample collection, sampling section, and the individual facility using pairwise PERMANOVA. Our results indicate that the composition of the microbiota did not vary significantly by month $(p>0.05$, Additional file 1 : 

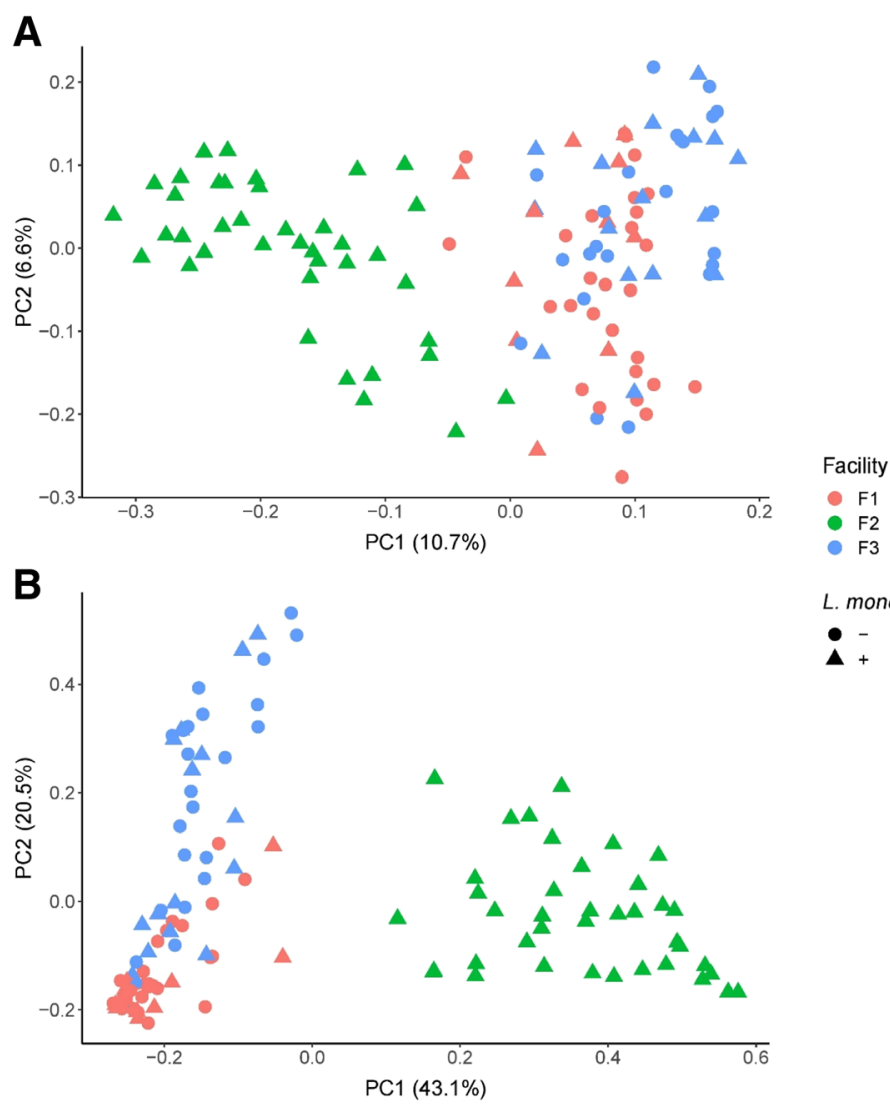

Fig. 5 Clustering of bacterial (a) and fungal (b) communities based on the UniFrac distances calculated using 16S rRNA V4 gene sequences for bacteria and ITS2 sequences for fungi. The colors red, green, and blue indicate samples from facilities F1, F2 and F3, respectively. The circles and triangles indicate samples positive (+) or negative $(-)$ for L. monocytogenes culture, respectively

Table S4). However, we found that different facilities and sections had a significant effect on the composition of microbiota $(p<0.003$; Table 2). Mycobiota composition was also not significantly different in different months (Additional file 1: Table S5), while it was significantly different in different facilities (Table 2). Moreover, when comparing samples from different processing sections, mycobiota in washing and fan-drying, as well as in fandrying and waxing sections was not significantly different $(p=0.057$ and 1.00 , respectively). However, mycobiota differed significantly between washing and waxing sections $(p=0.012$; Additional file 1: Table S3).

Table 2 Comparison of microbiota and mycobiota composition among different facilities

\begin{tabular}{|c|c|c|c|c|c|c|c|}
\hline Facility pair & $\mathrm{DF}^{\mathrm{a}}$ & Sum of squares & $F$ model & $R^{2 b}$ & $P$ value & $P$ adjusted & $\overline{S i g}$ \\
\hline \multicolumn{8}{|l|}{ Microbiota } \\
\hline F1 vs F2 & 1 & 2.788 & 7.664 & 0.092 & 0.001 & 0.003 & A \\
\hline F1 vs F3 & 1 & 1.525 & 3.904 & 0.049 & 0.001 & 0.003 & B \\
\hline F2 vs F3 & 1 & 3.259 & 8.983 & 0.106 & 0.001 & 0.003 & C \\
\hline \multicolumn{8}{|l|}{ Mycobiota } \\
\hline F1 vs F2 & 1 & 10.111 & 82.072 & 0.519 & 0.001 & 0.003 & A \\
\hline F1 vs F3 & 1 & 3.247 & 23.382 & 0.235 & 0.001 & 0.003 & B \\
\hline F2 vs F3 & 1 & 8.281 & 65.764 & 0.464 & 0.001 & 0.003 & C \\
\hline
\end{tabular}

${ }^{\mathrm{a}} \mathrm{DF}$ degree of freedom

${ }^{\mathrm{b}} R^{2} \mathrm{R}$ square

${ }^{\mathrm{c}} \mathrm{Sig}$, letter indicates significant difference between facility pairs 
Communities of environmental samples from facility F2 are predominated by Pseudomonadaceae and

\section{Dipodascaceae}

To explain the differential clustering of microbiota from samples collected in facility F2, we examined the taxonomic composition of the samples from different facilities (Fig. 6). Facilities F1, F2, and F3 contained 12, 8, and 14 unique bacterial families that were present at $10 \%$ or higher relative abundance (RA), respectively (Fig. 6). Families present in less than $10 \%$ relative abundance were grouped in a category "Other." The top three most abundant families found in facility F1 were Flavobacteriaceae (19.68\%), Moraxellaceae (11.93\%), and Weeksellaceae (10.27\%). High abundance of Pseudomonadaceae was found in facility F2 (41.97\%), where Flavobacteriaceae (18.27\%) and Xanthomonadaceae (7.69\%) were also relatively highly abundant. Facility F3 had highly abundant Weeksellaceae (13.22\%), Flavobacteriaceae (11.45\%) and both Burkholderiaceae and Moraxellaceae were present at $8.67 \%$. Pseudomonadaceae was identified as a predominant family in samples from facility F2 (41.97\% RA), which also had the highest occurrence of L. monocytogenes. Samples from facility F2 had significantly higher RA of Pseudomonadaceae compared to facilities F1 and F3 which contained $7.76 \%$ and $6.48 \%$ Pseudomonadaceae respectively $(p<0.0001)$. There was no significant difference in Pseudomonadaceae abundance between facilities F1 and F3 ( $p=$ 0.844 ). Another bacterial family that was frequently identified in high relative abundance in facility F2 was Flavobacteriaceae; however, it was frequently present in high relative abundance also in samples from facilities F1 and F3.

We further explored the difference in fungal communities among three monitored facilities by comparing mycobiota taxonomy at a family level (Fig. 7). The top three abundant families found in facility F1 were Aureobasidiaceae (30.04\%), Aspergillaceae (12.69\%), and Bulleribasidiaceae (9.50\%). Facility F2 had three highly abundant families, including Dipodascaceae (56.08\%), Trichosporonaceae (10.88\%), and Aspergillaceae (4.52\%), and the most abundant three fungal families found in facility F3 included Trichosporonaceae (30.63\%), Aureobasidiaceae (18.2\%), and Pleosporaceae (9.9\%). A higher abundance of Dipodascaceae was observed

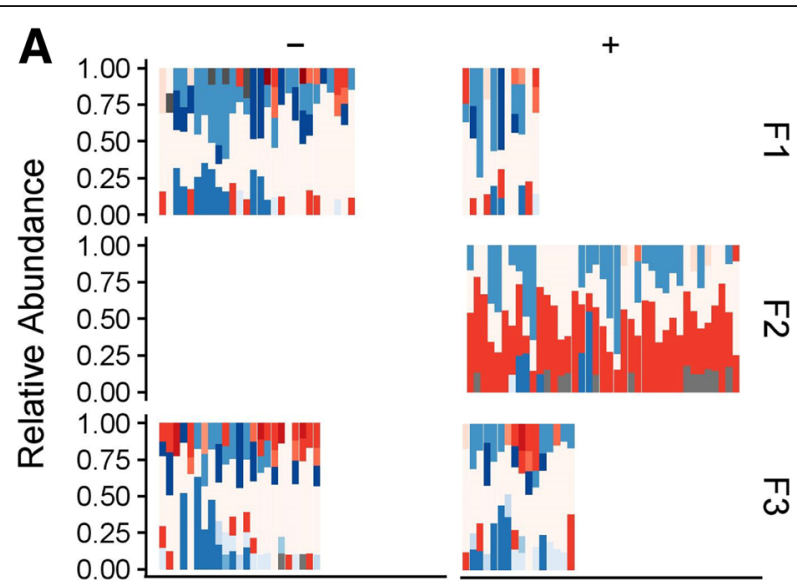

B

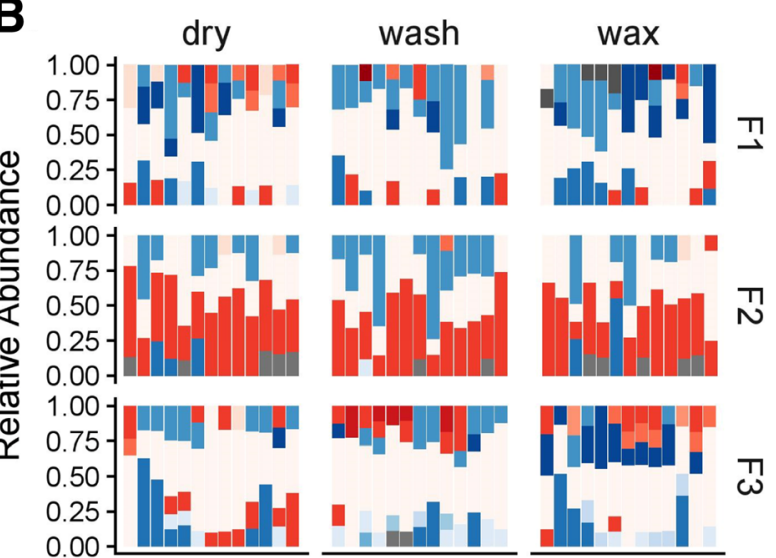

Family
Aeromonadaceae
Arcobacteraceae
Azospirillaceae
Bdellovibrionaceae
Burkholderiaceae
Caulobacteraceae
Chitinophagaceae
Enterobacteriaceae
Flavobacteriaceae
Moraxellaceae
Other
Pseudomonadaceae
Rhodobacteraceae
Sphingobacteriaceae
Sphingomonadaceae
uncultured
Weeksellaceae
Xanthomonadaceae

Fig. 6 Relative abundances of bacterial families in samples collected in three tree fruit packing facilities, shown by the presence (+) or absence (-) of L. monocytogenes culture in facilities F1, F2, and F3 (a) and in three sections (washing, fan drying, and waxing) within facilities (b). Families with relative abundance of less than $10 \%$ are shown under the category "Other." Each bar represents an individual sample 


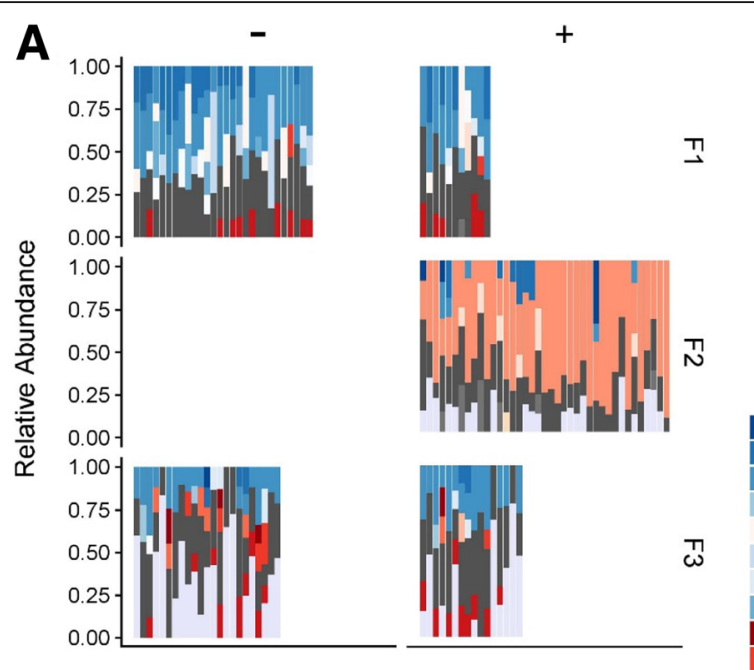

\section{Family}

Ascomycota unclassified

Aspergillaceae

Aureobasidiaceae

Botryosphaeriaceae

Bulleribasidiaceae

Ciliophora unclassified

Cladosporiaceae

Cucurbitariaceae

Didymellaceae

Didymosphaeriaceae

B

B $1.00-$ wash

Dipodascaceae

Fungi unclassified

Helotiales fam incertae sedis

Mycosphaerellaceae

Nectriaceae

Other

Phaeosphaeriaceae

Pleosporaceae

Saccharomycetales fam incerae sedis

仓ั
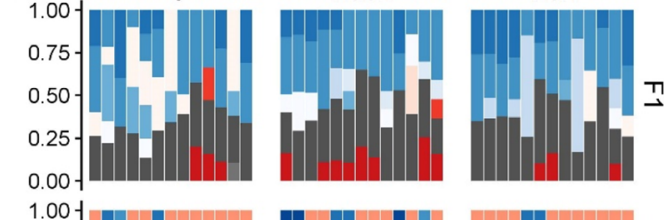

Trichosporonaceae

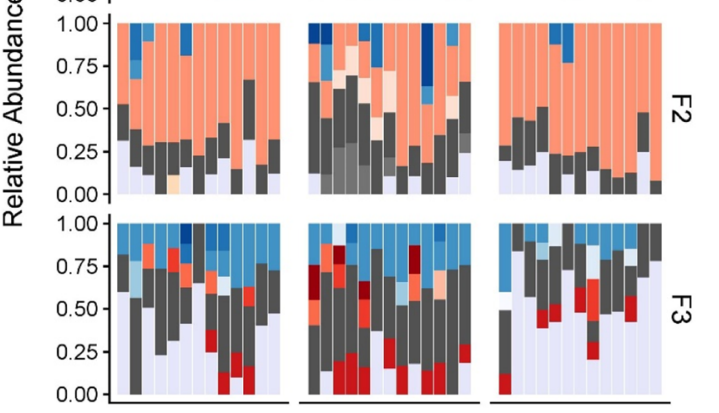

Fig. 7 Relative abundances of fungal families in samples collected in three tree fruit packing facilities, shown by the presence $(+)$ or absence $(-)$ of L. monocytogenes culture in facilities F1, F2, and F3 (a) and in three sections (washing, fan drying, and waxing) within facilities (b). Families with relative abundance less than $10 \%$ are shown under the category "Other." Each bar represents an individual sample

in facility F2, whereas it was present at very low RA in both facilities F1 $(0.93 \%)$ and F3 $(0.48 \%)(p<0.01)$. In contrast, Aureobasidiaceae was present at a relatively high level in facilities F1 and F3 and was rarely found in environmental samples of F2.

Since PICRUSt analyses were conducted on data obtained using Greengenes database, we compared the relative abundances of bacterial families determined based on Greengenes database with those determined based on SILVA database (Additional file 1: Table S6). The results showed that the differences in relative abundances were insignificant when all taxa identified using SILVA database were considered ( $p=0.975)$. Further, separate comparison of relative abundances of taxa with high relative abundance $(>0.0001)$ and low relative abundance $(<0.0001)$ confirmed insignificant differences ( $p=0.9675$ and $p=0.1389$, respectively) among results obtained based on Greengenes and SILVA databases.

\section{Network analysis of microbiota showed co-occurrence patterns between Pseudomonadaceae, \\ Enterobacteriaceae, and Rhizobiaceae}

In order to further explore the relationships between different microbial taxa, especially those that were highly abundant in F2, network analysis was carried out to investigate the co-occurrence or co-exclusion relationship of microbiota in tree fruit packing house built environment. Since facility F2 was previously identified as having the highest occurrence of L. monocytogenes, as well as having a relatively higher abundance of bacterial family Pseudomonadaceae, we sub-grouped the network to assess the interactions with a focus on the microbial families in direct relationship with Pseudomonadaceae. 
Families that co-occurred or co-excluded with Pseudomonadaceae were analyzed with all samples combined as well as with just samples from individual facilities. Both analyses were carried out to minimize potentially spurious relationships induced due to the significantly different composition of microbiota found among facilities. In facility F1, Pseudomonadaceae co-occurred only with Enterobacteriaceae, and Enterobacteriaceae was positively correlated with the occurrence of Rhizobiaceae (Fig. 8a). No co-occurrence or co-exclusion relationships between Pseudomonadaceae and other taxa have been found when samples from facility F2 were analyzed separately from other samples. Families Rhizobiaceae, Arcobacteraceae, Paludibacteraceae, and one Parcubacteria unclassified family were found to co-occur with Pseudomonadaceae in samples from facility F3 (Fig. 8b). A similar trend of the relationship was observed when samples from all facilities were analyzed together. In the network of all analyzed samples, Pseudomonadaceae was shown to have a significantly positive correlation with families Rhizobiaceae, Enterobacteriaceae, and Dysgonomonadace. On the other hand, Pseudomonadaceae had co-exclusionary relation with Spirosomaceae and Bdellovibrionaceae (Fig. 8c).

\section{PICRUSt predicted the differential abundance of bacterial taxa associated with certain functional categories in microbiota found in facility F2 compared to those found in facilities F1 and F3}

PICRUSt analysis was performed for functional profile predictions of microbiota based on 16S rRNA marker gene. Firstly, the absolute KEGG ortholog categories (KO) abundance in tier 1 functional categories were plotted for samples grouped by individual facility (Fig. 9a). Facility 2 microbiota samples showed a substantially higher abundance of identified $\mathrm{KO}$ in every category, compared to microbiota found in samples from facilities F1 and F3. We further calculated the relative abundance of tier 1 categories of the total absolute abundance in each facility (Fig. 9b). Since categories metabolism, environmental information processing, and cellular processes were considered to be more relevant to our environmental samples, we plotted each of these three categories separately. Microbiota showed a significant difference in functional category
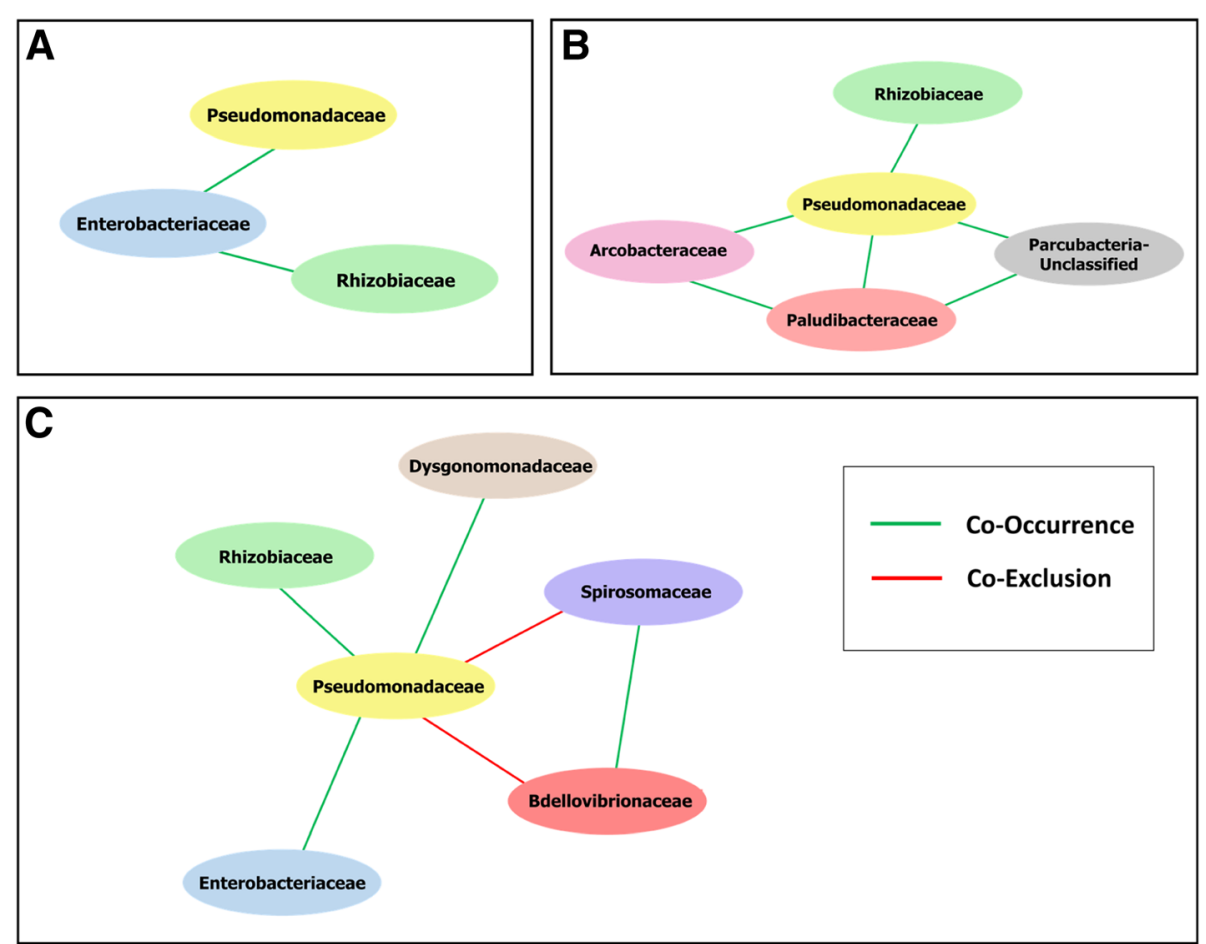

Fig. 8 Microbial networks indicating co-occurrence of microbial families in samples collected from three facilities F1, F2, and F3. Green edges represent positive relationship (co-occurrence) among families, whereas the red edges represent negative relationship (co-exclusion) between two connected nodes. Interaction network was sub-grouped with focus on Pseudomonadaceae, a bacterial family that predominated in microbiota of facility F2 that had the highest occurrence of L. monocytogenes. Interaction correlation between Pseudomonadaceae when only facility F1 samples were analyzed is shown in a panel (a). Interaction correlation between Pseudomonadaceae when only facility F3 samples were analyzed is shown in a panel (b). Interaction correlation between Pseudomonadaceae and other families when all samples are analyzed together is shown in a panel (c) 


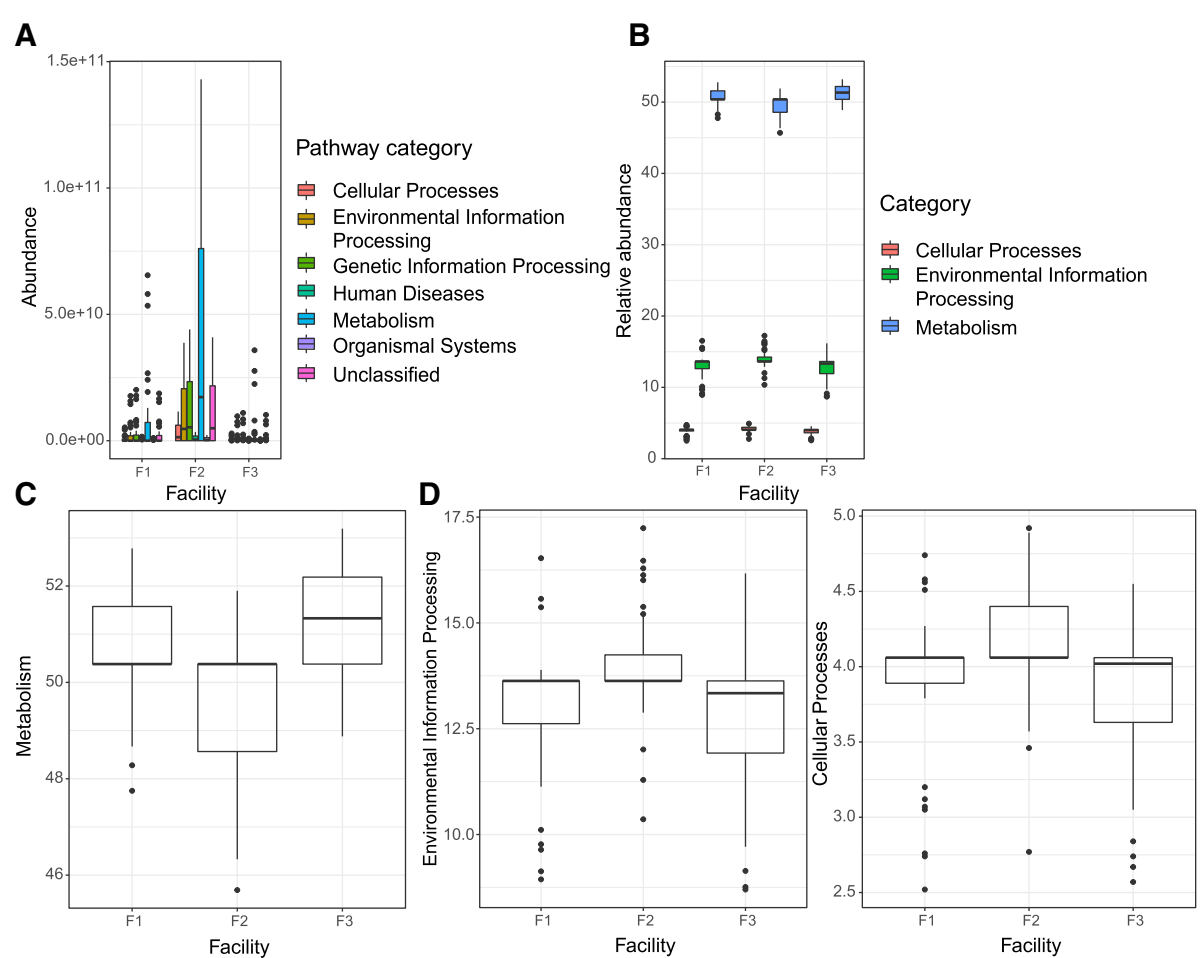

Fig. 9 Predicted functional profiles of microbiota in facilities F1, F2, and F3 derived from PICRUSt inference based on tier 1 KEGG ortholog categories (KO). Absolute abundance of tier 1 functional categories by facility is shown in a panel (a). Relative abundance of selected tier 1 functional categories by facility is shown in a panel (b). Relative abundance of predicted metabolism of microbiota by facility is shown in a panel (c). Relative abundance of Environmental information processing functional category in microbiota from three facilities is shown in a panel (d). Relative abundance of Cellular processes functional category in microbiota from three facilities is shown in a panel (e)

metabolism when F2 and F1 $(p=0.0016)$ as well as F2 and F3 $\left(p=8.1 \times 10^{-8}\right)$ were compared. There was no significant difference detected in the relative abundance of metabolism between F1 and F3 $(p=0.0522)$ (Fig. 9c). Comparing the environmental information processing profile, facility F2 again showed a significantly higher relative abundance compared to F1 $(p=0.0194)$ and F3 $(p=$ $0.0019)$. When cellular processes profile was compared in microbiota from different facilities, facility $2 \mathrm{KO}$ abundance in this category was significantly different from that in F1 $(p=0.0279)$ and F3 $(p=0.0016)$. The abundance of cellular processes $\mathrm{KO}$ in facilities $\mathrm{F} 1$ and $\mathrm{F} 3$ was not significantly different $(p=1.000)$.

\section{Discussion}

\section{Less sanitary conditions likely contributed to a higher} occurrence of $L$. monocytogenes in facility $\mathrm{F} 2$

Facility F2 had a significantly higher occurrence of L. monocytogenes compared to facilities F1 and F3. This may be explained by a different facility equipment set-up. For example, while both F1 and F3 were fully equipped, facility F2 did not install a dripping pan under the wet processing line to contain water and organic residues, including leaves, stems, and fruit pieces during processing. Water and organic residues therefore accumulated on the concrete floor underneath the conveyor where they potentially created a nutrient-rich environment suitable for microbial growth. The lack of a proper drainage system and poor cleaning and sanitizing practices may also have contributed to the unique microbiota in the built environment of the facility F2. The presence of cracks in the floor and between the equipment-floor junctions adds on the difficulties of cleaning, and it may promote the growth of microbial biofilm and persistence of L. monocytogenes contamination in these areas [52].

\section{Reduced diversity of microbial communities in facility F2} is indicative of the persistence of $L$. monocytogenes

Rarefaction curves indicated the lowest bacterial and fungal species richness in environmental samples from facility F2, while microbial richness was substantially higher in facilities F1 and F3. The microbiota and mycobiota rarefaction curves for samples collected in facilities F1 and F3 did not reach a plateau, indicating a considerable undiscovered diversity at a used sequencing depth. The indications of rarefaction curves were confirmed by low alpha diversity in samples from facility F2. Lower diversity in microbial species may result in less 
competition for nutrients among microorganisms as well as fewer inhibitory secondary metabolites produced by certain microorganisms [53]. Leriche and Carpentier found that a decreased adhesion was found for L. monocytogenes when it was co-cultured with Staphylococcus sciuri due to competition for nutrients [19]. Secondary metabolites, including bacteriocin-like compounds produced by isolated lactic acid bacteria, have also been shown to have antagonistic activity against $L$. innocua, a close relative which is commonly used to model the behavior of L. monocytogenes [54]. Production of enzymes that are lytic to cell wall is another mode of antimicrobial action found in yeast, including Candida guilliermondii and Candida oleophila that have been shown to secrete hydrolytic enzymes that can be used to control Botrytis cinerea on tomato plants [55]. Lower alpha diversity in F2, as well as distinct clustering of microbiota from F2 based on the beta diversity, indicates the ecologically distinct character of facility F2 that potentially supports the growth and/or persistence of L. monocytogenes. Based on our collected data, it is not possible to speculate whether the lower microbial diversity in facility F2 led to the persistence of $L$. monocytogenes, or the establishment of $L$. monocytogenes led to reduced alpha diversity and distinct microbial ecology compared to facilities F1 and F3. It is noteworthy, though, that facilities F1 and F3 both have a similar microbiota composition despite different locations.

\section{Pseudomonadaceae and Dipodascaceae predominate in facility F2 and represent microorganisms that are known biofilm formers and indicators of unhygienic conditions, respectively}

When comparing the identified bacterial families and their corresponding relative abundances obtained based on Greengenes database and SILVA database, inconsistencies were mainly found among relatively low abundant taxa. However, statistical comparison showed that analysis based on taxonomic classification using different databases did not result in significantly different composition on a family level. Pseudomonadaceae was predominant in the environment of the facility F2 with persistent $L$. monocytogenes contamination and is comprised of known biofilm formers, including Azomonas, Azorhizophilus, Azotobacter, Mesophilobacter, and Pseudomonas [56]. Pseudomonas species are known for their ability to adapt and thrive in the food processing environment [57]. They may have a growth advantage in a cold and humid food processing environment that allows them to outcompete a number of other microorganisms because they can grow on a variety of nutrient sources, tolerate low temperatures, and form a biofilm [58]. These properties make Pseudomonas successful in colonization of environmental surfaces. Previous research has demonstrated that $L$. monocytogenes adheres to surfaces substantially better when co-cultured with Pseudomonas fragi. Biofilm-forming Pseudomonas could shelter $L$. monocytogenes and enhance its survivability under recurrent cleaning and sanitizing treatments [59]. This protection is conferred by an EPS that provides a physical barrier that reduces diffusion of chemicals into the core of the biofilm structure, as well as provides nutrients for bacterial growth. L. monocytogenes can survive for a longer period of time when co-cultured with $P$. pudida even with no added nutrients [18]. These previous findings and our observation of significantly higher relative abundance of Pseudomonadaceae in facility F2 led us to hypothesize that biofilm formation may support $L$. monocytogenes persistence in this facility. Further studies investigating the mechanisms of microbial interactions in microbiota in $\mathrm{F} 2$ are hence warranted.

Another, fungal, family Dipodascaceae was the predominant family detected in facility F2 mycobiota, whereas it was low-abundant or absent in facilities F1 and F3. Dipodascaceae are white filamentous fungi belonging to the order of Saccharomycetales. Microorganisms belonging to this family have been previously shown to have an ability to elevate granular sludge lipid accumulation due to the production of linoleic acid [60]. For example, species Geotrichum candidum, referred to as "machinery mould," is a common species belonging to Dipodascaceae family and is ubiquitously present in the natural environment [61]. It is a plant pathogen that causes post-harvest sour-rot spoilage of fruit and vegetable during storage [61]. G. candidum can form a white slime on surfaces that have contact with produce residues and can be readily removed with good sanitation practices [62]. Therefore, it has been historically used as a fungal indicator of poor hygienic status in fruit and vegetable processing plants that are associated with inadequate sanitation [63]. Although there is no direct information indicating that Dipodascaceae could promote the survival of L. monocytogenes, the presence of G. candidum does indicate the overall poor hygienic status of facility F2. With reduced hygienic status, the risk of foodborne pathogen contamination and colonization in the facility increases [64].

The unhygienic environmental conditions in facility F2 may have shaped the microbial communities in the environment toward a predominance of Pseudomonadaceae and Dipodascaceae families, as well as L. monocytogenes. Furthermore, inadequate cleaning and sanitizing of the facility presents an increased risk for cross-contamination of produce from the produce processing environment.

Although L. monocytogenes is not able to grow on apples, the pathogen can survive on the surface of the fresh fruit [65]. Given that apples are consumed raw, their contamination with $L$. monocytogenes presents a health risk for certain groups of consumers, such as elderly and immunocompromised that can get sick by ingestion of as 
few as 0.1 million to 10 million CFU of $L$. monocytogenes, based on the risk assessment of $L$. monocytogenes in Canada [66]. FAO/WHO estimated the risk of L. monocytogenes based on epidemiological data later in 2014, and predicted that exposure to $10,000 \mathrm{~L}$. monocytogenes cells results in one case of listeriosis infection in every 20 million people [67]. However, based on two recent outbreaks in the USA and Europe, which involved immunocompromised patients and possibly hypervirulent strains, the probability of infection after consumption of one cell of $L$. monocytogenes was estimated to be almost 100,000 times higher than that estimated by FAO/WHO in 2004 based on epidemiologic data of patients from all susceptible population groups [68].

In cases when fresh apples are coated with caramel and punctured with a wooden stick, the risk increases, as this creates microenvironments suitable for pathogen growth [9]. Better understanding of the interactions, growth, and/or persistence of $L$. monocytogenes in natural microbiomes, including microbiome biofilms, is needed. Improved knowledge in this area could lay a foundation for precise optimization of cleaning and sanitizing procedures to effectively reduce $L$. monocytogenes in built environments through manipulation of microbial interactions.

\section{Co-occurrence and co-exclusion relationships between Pseudomonadaceae and other bacterial families}

We applied network analysis to describe the co-occurrence and co-exclusion relationships between Pseudomonadaceae and other bacterial families that may contribute to $L$. monocytogenes survival and persistence in apple packing house environments. The co-occurrence of Enterobacteriaceae, Pseudomonas, and Rhizobiaceae in the environment of the facility 2 is noteworthy, as they are related due to the ease of genetic material exchange [69]. For example, an antibiotic-resistant $\mathrm{R}$ factor is inter-transferable among genera comprising Enterobacteriaceae, Pseudomonas, and Rhizobiaceae [70]. Another study based on Adansonian analysis has shown that members of Rhizobiaceae are highly related to strains in Escherichia coli and Enterobacter aerogenes, while genera Rhizobium and Phytomyxa from the family of Rhizobiaceae are closely related to members in Pseudomonadaceae family [71]. Besides genetic relatedness, mutualism based on metabolites has also been found between Pseudomonas aeruginosa PA14 and Enterobacter aerogenes, where the co-culture of the two organisms had at least 14-fold increase in current density compared to either species alone [72]. In contrast, Pseudomonadaceae and Bdellovibrionaceae were found to co-exclude each other when all samples were analyzed together. Bdellovibrio, a genus from the family of Bdellovibrionaceae that was shown to negatively co-occur with Pseudomonadaceae in our study, has been previously shown to have predatory activity toward Gram-negative bacteria, especially Escherichia coli and Pseudomonas (i.e., Pseudomonas syringae [73] and Pseudomonas aeruginosa [74]). Furthermore, Bdellovibrio bacteriovorus has been shown to reduce existing E. coli and P. fluorescence biofilm biomass, and Bdellovibriotreated biofilm has been more easily washed off compared to a control [75]. Pseudomonas aeruginosa is also considered as one of B. bacteriovorus' prey [76]. Limited information is available about characteristics of microorganisms belonging to the family Spriosomaceae, another family that was negatively co-occurring with Pseudomonadaceae. Network analysis based on fungal families identified a fungal family Dipodascaceae which was found in high relative abundance in facility F2 as negatively associated with a number of other fungal families, and positively associated only with an undefined fungal family Saccharomycetalesfam-Incertae-sedis (Additional file 1: Figure S1). Given that many fungal OTUs were classified as unknown and were hence not further classified into known fungal families, these results need to be interpreted with caution. Network analysis of fungal and bacterial families combined showed similar relationship when they were analyzed separately, where bacterial family Pseudomonadaceae was identified as positively associated with the occurrence of Enterobacteriaceae, Rhizobiaceae, and Dysgonomonadaceae, and fungal family Dipodascaceae was found to be positively associated with the presence of "Saccharomycetales-fam-Incertaesedis," and negatively associated with the occurrence of "unclassified-Ciliophora" (Additional file 1: Figure S2). Our network analysis indicates a potential mechanism underlying the high prevalence of certain bacterial families in the processing environments. The positive relationship may indicate the mutualistic interactions, whereas the negative correlation may suggest the inhibitory interactions among microbiota. Nevertheless, these hypothesized correlations need to be further studied experimentally.

\section{Predicted bacterial functional profiles and putative underlying mechanisms}

Samples collected in facility F2 had a substantially higher number of identified KOs compared to samples from F1 and F3, indicating a higher number of functional units were characteristic of microbiota detected in facility F2. This is contradictory to what has been found in other studies where they speculated a decline in functionality in less diverse microbiota communities. However, this may also be a result of urbanization-introduced biotic similarity [77], as some of the other studies have shown that low functionality can also be found in highly diverse bacterial communities, as a result of functional redundancy [78].

Facility F2 microbiota had a higher relative abundance of $\mathrm{KO}$ in the metabolism and cellular processes category, whereas it had lower relative $\mathrm{KO}$ in the environmental 
information processing category compared to the microbiota from facilities F1 and F3. This is consistent with our hypothesis that led to further analyses of sub-tier categories. Category metabolism includes the production of secondary metabolites, which we proposed earlier as one of the potential mechanisms through which persistent colonization of L. monocytogenes is inhibited in facilities F1 and F3. On the other hand, the bacterial secretion system which belongs to tier 1 category environmental information processing, plays a crucial role in the $P$. aeruginosa biofilm formation [79]. Cell communication, as one of the sub-categories of cellular processes, may also help explain the presence of the biofilm-formers in facility F2 since quorum sensing and bacterial biofilm formation are highly linked [80]. Moreover, previous studies have shown that type VI secretion systems (T6SSs) may be involved in the biofilm formation in Gramnegative bacteria including Pseudomonas spp. [81]. Although a clear association between the diversity and functionality of native microorganisms present in the built tree fruit processing facility environment needs to be further investigated to validate these functional predictions, our results provide foundational information that may be used to develop targeted study designs to help elucidate the impact of built environment microbial communities on the survival, growth, and persistence of pathogenic microorganisms such as L. monocytogenes in food processing facilities. This is particularly important as the use of new biocontrol products is gaining in popularity and needs to be carefully assessed prior to application to avoid adverse outcomes.

\section{Outlook, novelty, and importance}

When designing the cleaning and sanitizing protocols, it is important to consider the microbial landscape in the built environment of food processing facilities. It has been shown that planktonic L. monocytogenes could be reduced by 5 logs within $30 \mathrm{~s}$ of treatment, whereas the only weak effect was observed in attached cells even after an extended treatment time [82]. The complexity of in-house microbiota community is likely to reduce the effectiveness of disinfectants, and bacteria such as Pseudomonadaceae may help foodborne pathogen such as L. monocytogenes in survival and persistence in food processing environments and thus increase the food safety and public health risk. Our longitudinal survey of tree fruit packinghouse environmental microbiota suggests that the composition and diversity of microorganism found in food processing environments is associated with persisting L. monocytogenes contamination. Our study provides a data baseline needed for further in-depth investigation of microbial interactions between non-pathogenic and pathogenic microorganisms found in food processing environments. Further research in the area may lead to the optimization of pathogen control strategies and the development of novel biocontrol methods to complement physical and chemical interventions and improve food safety.

\section{Additional file}

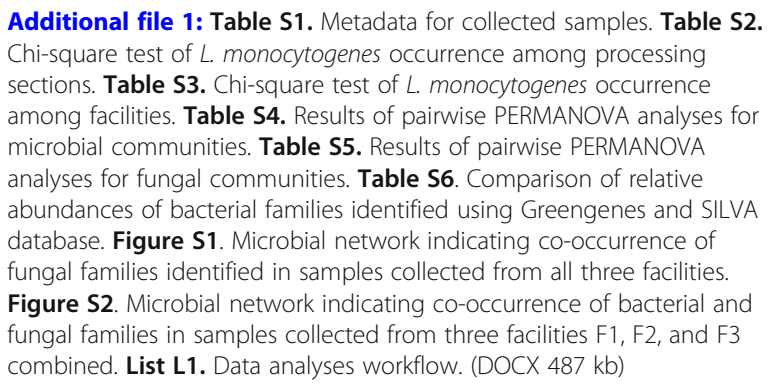

\section{Abbreviations}

ANOVA: Analysis of variance; BAM: Bacteriological analytical manual; BLEB: Buffered Listeria enrichment broth; DNA: Deoxyribonucleic acid; FDA: Food and Drug Administration; iap: Invasion associated protein; ITS: Internal transcribed spacer; KEGG: Kyoto Encyclopedia of Genes and Genomes; LM: Listeria monocytogenes; MPN: Most probable number; OTU: Operational taxonomic unit; PCOA: Principal coordinates analysis; PCR: Polymerase chain reaction; PERMANOVA: Permutational multivariate analysis of variance; PICRUSt: Phylogenetic Investigation of Communities by Reconstruction of Unobserved States; RA: Relative abundance;

RNA: Ribonucleic acid; TSAYE: Trypticase soy agar with yeast extract

\section{Acknowledgements}

We thank Tobin Simonetti for valuable assistance during experimental procedure design, as well as Dr. Yasser El-Manzalawy for guiding T. C. in network analysis. Sequencing was carried out using a MiSeq instrument funded by the U.S. Food and Drug Administration grant number 1U18FD006222-01 to Dr. Edward G. Dudley for support of GenomeTrakr in Pennsylvania.

\section{Authors' contributions \\ XT collected and analyzed the samples, carried out bioinformatics and statistical data analyses, and wrote the manuscript. TC carried out bioinformatics and statistical data analyses. Y C and DM contributed to the study design and revised the manuscript. LFL conceived the study, enabled access to sampling sites, provided pilot microbiological data, and revised the manuscript. JK conceived the study, guided the bioinformatics and statistical data analyses, and co-wrote the manuscript. All authors read and approved the final manuscript.}

\section{Funding}

J. K. and L. F. L. were supported by the USDA National Institute of Food and Agriculture Hatch Appropriations under projects \#PEN04646 and \#PEN04666, accession \#1015787, respectively. The project was supported by the Center for Food Safety and Applied Nutrition-Food and Drug Administration, grant-HHSF223201610124C.

\section{Availability of data and material \\ Amplicon sequencing data from this study were deposited in NCBI under BioProject accession number PRJNA527988.}

\section{Ethics approval and consent to participate \\ Not applicable.}

\section{Consent for publication}

Not applicable.

\section{Competing interests}

The authors declare that they have no competing interests.

\section{Author details}

'Department of Food Science, The Pennsylvania State University, University Park, PA 16802, USA. ${ }^{2}$ Microbiome Center, Huck Institutes of the Life Sciences, The Pennsylvania State University, University Park, PA 16802, USA. ${ }^{3}$ Center for Food Safety and Applied Nutrition, Food and Drug Administration, College Park, MD 20740, USA. 
Received: 26 April 2019 Accepted: 29 July 2019

\section{Published online: 21 August 2019}

\section{References}

1. Swaminathan B, Gerner-Smidt P. The epidemiology of human listeriosis. Microbes Infect. 2007;9:1236-43.

2. Gombas DE, Chen Y, Clavero RS, Scott VN. Survey of Listeria monocytogenes in ready-to-eat foods. J Food Prot. 2003;66:559-69.

3. Lovett J, Francis DW, Hunt JM. Listeria monocytogenes in raw milk: detection, incidence, and pathogenicity. J Food Prot. 1987;50:188-92.

4. Multistate outbreak of listeriosis linked to packaged salads produced at springfield, Ohio dole processing facility | Listeria | CDC. 2018. https://www.cdc. gov/listeria/outbreaks/bagged-salads-01-16/index.html. Accessed 20 Feb 2019.

5. Zhu Q, Gooneratne R, Hussain MA. Listeria monocytogenes in fresh produce: outbreaks, prevalence and contamination levels. Foods. 2017;6. https://doi.org/10.3390/foods6030021.

6. Multistate outbreak of listeriosis linked to commercially produced, prepackaged caramel apples made from Bidart Bros. Apples | Listeria | CDC. 2018. https://www.cdc.gov/listeria/outbreaks/caramel-apples-12-14/index. html. Accessed 20 Feb 2019.

7. Marus JR. Notes from the Field: outbreak of Listeriosis likely associated with prepackaged caramel apples-United States, 2017. MMWR Morb Mortal Wkly Rep. 2019;68. https://doi.org/10.15585/mmwr.mm6803a5.

8. Government of Canada CFIA. Sliced apples and products containing sliced apples recalled due to Listeria monocytogenes. 2015. http://www.inspection. gc.ca/about-the-cfia/newsroom/food-recall-warnings/complete-listing/2015042 9b/eng/1430375161334/1430375167258. Accessed 20 Feb 2019.

9. Glass KA, Golden MC, Wanless BJ, Bedale W, Czuprynski C. Growth of Listeria monocytogenes within a caramel-coated apple microenvironment. mBio. 2015;6:e01232-15.

10. Chavant P, Gaillard-Martinie B, Hébraud M. Antimicrobial effects of sanitizers against planktonic and sessile Listeria monocytogenes cells according to the growth phase. FEMS Microbiol Lett. 2004;236:241-8.

11. Lundén J, Autio T, Markkula A, Hellström S, Korkeala H. Adaptive and crossadaptive responses of persistent and non-persistent Listeria monocytogenes strains to disinfectants. Int J Food Microbiol. 2003:82:265-72.

12. Pan Y, Breidt F, Kathariou S. Resistance of Listeria monocytogenes biofilms to sanitizing agents in a simulated food processing environment. Appl Env Microbiol. 2006;72:7711-7.

13. Taormina PJ, Beuchat LR. Survival of Listeria monocytogenes in commercial food-processing equipment cleaning solutions and subsequent sensitivity to sanitizers and heat. J Appl Microbiol. 2002:92:71-80.

14. Beuchat LR, Adler BB, Lang MM. Efficacy of chlorine and a peroxyacetic acid sanitizer in killing Listeria monocytogenes on iceberg and Romaine lettuce using simulated commercial processing conditions. J Food Prot. 2004;67:1238-42.

15. Bokulich NA, Lewis ZT, Boundy-Mills K, Mills DA. A new perspective on microbial landscapes within food production. Curr Opin Biotechnol. 2016;37:182-9.

16. Doyle CJ, OToole PW, Cotter PD. Metagenome-based surveillance and diagnostic approaches to studying the microbial ecology of food production and processing environments. Environ Microbiol. 2017;19:4382-91.

17. Giaouris E, Heir E, Desvaux M, Hébraud M, Møretrø T, Langsrud S, Doulgeraki A, Nychas GJ, Kačániová M, Czaczyk K, Ölmez H, Simões M. Intra- and interspecies interactions within biofilms of important foodborne bacterial pathogens. Front Microbiol. 2015;20;6:841. https://doi.org/10.3389/fmicb.2 015.00841

18. Hassan AN, Birt DM, Frank JF. Behavior of Listeria monocytogenes in a Pseudomonas putida biofilm on a condensate-forming surface. J Food Prot. 2004;67:322-7

19. Leriche V, Carpentier B. Limitation of adhesion and growth of Listeria monocytogenes on stainless steel surfaces by Staphylococcus sciuri biofilms. J Appl Microbiol. 2000;88:594-605.

20. Carpentier B, Chassaing D. Interactions in biofilms between Listeria monocytogenes and resident microorganisms from food industry premises. Int J Food Microbiol. 2004;97:111-22.

21. Kunze B, Reck M, Dötsch A, Lemme A, Schummer D, Irschik H, et al. Damage of Streptococcus mutans biofilms by carolacton, a secondary metabolite from the myxobacterium Sorangium cellulosum. BMC Microbiol. 2010;10:199

22. Liu Y, Zhang H, Wu C, Deng W, Wang D, Zhao G, et al. Molecular analysis of dominant species in Listeria monocytogenes-positive biofilms in the drains of food processing facilities. Appl Microbiol Biotechnol. 2016;100:3165-75.
23. Stellato G, Storia AL, Filippis FD, Borriello G, Villani F, Ercolini D. Overlap of spoilage-associated microbiota between meat and the meat processing environment in small-scale and large-scale retail distributions. Appl Env Microbiol. 2016:82:4045-54.

24. Fox EM, Solomon K, Moore JE, Wall PG, Fanning S. Phylogenetic profiles of in-house microflora in drains at a food production facility: comparison and biocontrol implications of Listeria-positive and -negative bacterial populations. Appl Environ Microbiol. 2014;80:3369-74.

25. Ho AJ, Lappi VR, Wiedmann M. Longitudinal monitoring of Listeria monocytogenes contamination patterns in a farmstead dairy processing facility. J Dairy Sci. 2007;90:2517-24.

26. Kabuki DY, Kuaye AY, Wiedmann M, Boor KJ. Molecular subtyping and tracking of Listeria monocytogenes in Latin-style fresh-cheese processing plants. J Dairy Sci. 2004;87:2803-12.

27. Beuchat LR. Pathogenic microorganisms associated with fresh produce. J Food Prot. 1996;59:204-16

28. Berger CN, Sodha SV, Shaw RK, Griffin PM, Pink D, Hand P, et al. Fresh fruit and vegetables as vehicles for the transmission of human pathogens. Environ Microbiol. 2010;12:2385-97.

29. Gu G, Ottesen A, Bolten S, Wang L, Luo Y, Rideout S, et al. Impact of routine sanitation on the microbiomes in a fresh produce processing facility. Int J Food Microbiol. 2019;294:31-41.

30. Naing L, Winn T, Rusli BN. MEDICAL STATISTICS Practical issues in calculating the sample size for prevalence studies. Archives of Orofacial Sciences. 2006;1:9-14.

31. FDA. Laboratory methods-BAM: detection and enumeration of Listeria monocytogenes. 2017. https://www.fda.gov/food/foodscienceresearch/ laboratorymethods/ucm071400.htm. Accessed 11 Dec 2018.

32. Chen Y, Knabel SJ. Multiplex PCR for simultaneous detection of bacteria of the genus Listeria, Listeria monocytogenes, and major serotypes and epidemic clones of L. monocytogenes. Appl Environ Microbiol. 2007;73:6299-304.

33. FDA. Laboratory methods-BAM Appendix 2: most probable number from serial dilutions. 2017. https://www.fda.gov/Food/FoodScienceResearch/ LaboratoryMethods/ucm 109656.htm. Accessed 31 Jan 2019.

34. Tringe SG, Zhang T, Liu X, Yu Y, Lee WH, Yap J, et al. The airborne metagenome in an indoor urban environment. PloS One. 2008;3:e1862.

35. Thompson LR, Sanders JG, McDonald D, Amir A, Ladau J, Locey KJ, et al. A communal catalogue reveals Earth's multiscale microbial diversity. Nature. 2017:551:457-63.

36. Nordberg H, Cantor M, Dusheyko S, Hua S, Poliakov A, Shabalov I, et al. The genome portal of the Department of Energy Joint Genome Institute: 2014 updates. Nucleic Acids Res. 2014;42(Database issue):D26-31.

37. 16s-metagenomic-library-prep-guide-15044223-b.pdf. https://support. illumina.com/documents/documentation/chemistry_documentation/16s/16 s-metagenomic-library-prep-guide-15044223-b.pdf. Accessed 11 Dec 2018.

38. Schloss PD, Westcott SL, Ryabin T, Hall JR, Hartmann M, Hollister EB, et al. Introducing mothur: open-source, platform-independent, communitysupported software for describing and comparing microbial communities. Appl Environ Microbiol. 2009;75:7537-41.

39. Kozich JJ, Westcott SL, Baxter NT, Highlander SK, Schloss PD. Development of a dual-index sequencing strategy and curation pipeline for analyzing amplicon sequence data on the MiSeq Illumina sequencing platform. Appl Env Microbiol. 2013;79:5112-20.

40. Quast C, Pruesse E, Yilmaz P, Gerken J, Schweer T, Yarza P, et al. The SILVA ribosomal RNA gene database project: improved data processing and web-based tools. Nucleic Acids Res. 2013;41(Database issue):D590-6.

41. Kõljalg U, Nilsson RH, Abarenkov K, Tedersoo L, Taylor AFS, Bahram M, et al. Towards a unified paradigm for sequence-based identification of fungi. Mol Ecol. 2013;22:5271-7.

42. McMurdie PJ, Holmes S. phyloseq: an R package for reproducible interactive analysis and graphics of microbiome census data. PLoS One. 2013;8(4): e61217. https://doi.org/10.1371/journal.pone.0061217.

43. Wickham H. ggplot2: elegant graphics for data analysis: Springer; 2016.

44. Arbizu M. pairwiseAdonis: pairwise multilevel comparison using Adonis: $\mathrm{R}$ package version 0.3; 2019. https://github.com/pmartinezarbizu/ pairwiseAdonis. Accessed 4 Aug 2019.

45. Shannon P, Markiel A, Ozier O, Baliga NS, Wang JT, Ramage D, et al. Cytoscape: a software environment for integrated models of biomolecular interaction networks. Genome Res. 2003;13:2498-504.

46. Faust $K$, Raes J. CoNet app: inference of biological association networks using Cytoscape. F1000 Research. 2016;5. https://doi.org/10.12688/f1 000research.9050.2. 
47. Wiese R, Eiglsperger M, Kaufmann M. yFiles—visualization and automatic layout of Graphs. In: Jünger M, Mutzel P, editors. Graph Drawing Software. Berlin, Heidelberg: Springer Berlin Heidelberg; 2004. p. 173-91. https://doi. org/10.1007/978-3-642-18638-7_8

48. DeSantis TZ, Hugenholtz P, Larsen N, Rojas M, Brodie EL, Keller K, et al. Greengenes, a chimera-checked 16S rRNA gene database and workbench compatible with ARB. Appl Env Microbiol. 2006;72:5069-72.

49. Langille MGl, Zaneveld J, Caporaso JG, McDonald D, Knights D, Reyes JA, et al. Predictive functional profiling of microbial communities using 16S rRNA marker gene sequences. Nat Biotechnol. 2013;31:814-21.

50. Huttenhower. Galaxy. http://huttenhower.sph.harvard.edu/galaxy/. Accessed 24 Feb 2019.

51. Kanehisa M, Goto S. KEGG: kyoto encyclopedia of genes and genomes. Nucleic Acids Res. 2000;28:27-30.

52. Ferreira $V$, Wiedmann $M$, Teixeira $P$, Stasiewicz MJ. Listeria monocytogenes persistence in food-associated environments: epidemiology, strain characteristics, and implications for public HEALTH. J Food Prot. 2014;77:150-70.

53. Giaouris E, Heir E, Hébraud M, Chorianopoulos N, Langsrud S, Møretrø T, et al. Attachment and biofilm formation by foodborne bacteria in meat processing environments: Causes, implications, role of bacterial interactions and control by alternative novel methods. Meat Sci. 2014:97:298-309.

54. Ammor S, Tauveron G, Dufour E, Chevallier I. Antibacterial activity of lactic acid bacteria against spoilage and pathogenic bacteria isolated from the same meat small-scale facility: 1—screening and characterization of the antibacterial compounds. Food Control. 2006:17:454-61.

55. Saligkarias ID, Gravanis FT, Epton HAS. Biological control of Botrytis cinerea on tomato plants by the use of epiphytic yeasts Candida guilliermondii strains 101 and US 7 and Candida oleophila strain 1-182: II. a study on mode of action. Biol Control. 2002;25:151-61.

56. Drobish AM, Emery BD, Whitney AM, Lauer AC, Metcalfe MG, McQuiston JR. Oblitimonas alkaliphila gen. nov., sp. nov., in the family Pseudomonadaceae, recovered from a historical collection of previously unidentified clinical strains. Int J Syst Evol Microbiol. 2016;66:3063-70.

57. Rossi C, Serio A, Chaves-López C, Anniballi F, Auricchio B, Goffredo E, et al. Biofilm formation, pigment production and motility in Pseudomonas spp. isolated from the dairy industry. Food Control. 2018:86:241-8.

58. Sterniša M, Klančnik A, Možina SS. Spoilage Pseudomonas biofilm with Escherichia coli protection in fish meat at $5{ }^{\circ} \mathrm{C}$. J Sci Food Agric. 2019;0. doi: https://doi.org/10.1002/jsfa.9703.

59. Puga CH, Dahdouh E, SanJose C, Orgaz B. Listeria monocytogenes colonizes Pseudomonas fluorescens biofilms and induces matrix over-production. Front Microbiol. 2018;9. https://doi.org/10.3389/fmicb.2018.01706.

60. Li M, Li A, Sun Q, Jiang X, Chen S. Enhancement of biodiesel production by cultivating Dipodascaceae moderated-filamentous granular sludge with sugar-containing wastewater. Int Biodeterior Biodegrad. 2016;110:38-45.

61. Thornton CR, Slaughter DC, Davis RM. Detection of the sour-rot pathogen Geotrichum candidum in tomato fruit and juice by using a highly specific monoclonal antibody-based ELISA. Int J Food Microbiol. 2010;143:166-72.

62. Pitt Jl, Hocking AD. Fungi and Food Spoilage: Springer Science \& Business Media; 2009.

63. An Evaluation of the Role of Microbiological Criteria for Foods and Food Ingredients. National Academies; 1985.

64. Muhterem-Uyar M, Dalmasso M, Bolocan AS, Hernandez M, Kapetanakou AE, Kuchta T, et al. Environmental sampling for Listeria monocytogenes control in food processing facilities reveals three contamination scenarios. Food Control. 2015;51:94-107.

65. Smith A, Moorhouse E, Monaghan J, Taylor C, Singleton I. Sources and survival of Listeria monocytogenes on fresh, leafy produce. J Appl Microbiol. 2018;125:930-42.

66. Bortolussi R. Listeriosis: a primer. CMAJ. 2008;179:795-7.

67. FAO/WHO. Risk Assessment of Listeria monocytogenes in Ready-to-eat Foods: Technical Report. Food \& Agriculture Org, 2004. http://www.fao. org/3/y5394e/y5394e00.htm. Accessed 5 Aug 2019

68. Pouillot R, Klontz KC, Chen Y, Burall LS, Macarisin D, Doyle M, et al. Infectious dose of Listeria monocytogenes in outbreak linked to ice cream, United States, 2015. Emerg Infect Dis. 2016;22:2113-9.

69. Sanderson KE. Genetic Relatedness in the Family Enterobacteriaceae. Annu Rev Microbiol. 1976;30:327-49.

70. Datta N, Hedges RW, Shaw EJ, Sykes RB, Richmond MH. Properties of an R Factor from Pseudomonas aeruginosa. J Bacteriol. 1971;108:1244-9.

71. Moffett ML, Colwell RR. Adansonian Analysis of the Rhizobiaceae. J Gen Microbiol. 1968;51:245-66.
72. Venkataraman A, Rosenbaum MA, Perkins SD, Werner JJ, Angenent LT. Metabolite-based mutualism between Pseudomonas aeruginosaPA14 and Enterobacter aerogenes enhances current generation in bioelectrochemical systems. Energy Environ Sci. 2011:4:4550-9.

73. Barel G, Sirota A, Volpin H, Jurkevitch E. Fate of predator and prey proteins during growth of Bdellovibrio bacteriovorus on Escherichia coli and Pseudomonas syringae Prey. J Bacteriol. 2005;187:329-35.

74. lebba V, Totino V, Santangelo F, Gagliardi A, Ciotoli L, Virga A, et al. Bdellovibrio bacteriovorus directly attacks Pseudomonas aeruginosa and Staphylococcus aureus Cystic fibrosis isolates. Front Microbiol. 2014;5. https://doi.org/10.3389/fmicb.2014.00280.

75. Kadouri D, O'Toole GA. Susceptibility of Biofilms to Bdellovibrio bacteriovorus Attack. Appl Env Microbiol. 2005;71:4044-51.

76. Pantanella F, lebba V, Mura F, Dini L, Totino V, Neroni B, et al. Behaviour of Bdellovibrio bacteriovorus in the presence of Gram-positive Staphylococcus aureus. New Microbiol. 2018;41:145-52.

77. Staley C, Gould TJ, Wang P, Phillips J, Cotner JB, Sadowsky MJ. Core functional traits of bacterial communities in the Upper Mississippi River show limited variation in response to land cover. Front Microbiol. 2014;5. https://doi.org/10.3389/fmicb.2014.00414.

78. Shade A, Handelsman J. Beyond the Venn diagram: the hunt for a core microbiome. Environ Microbiol. 2012;14:4-12

79. KEGG. KEGG PATHWAY: Biofilm formation - Pseudomonas aeruginosa. https://www.genome.jp/kegg-bin/show_pathway?map=ko02025\&show_ description=show. Accessed 5 Mar 2019.

80. Nadell CD, Xavier JB, Levin SA, Foster KR. The evolution of quorum sensing in bacterial biofilms. PLOS Biol. 2008;6:e14.

81. Gallique M, Bouteiller M, Merieau A. The Type VI Secretion System: A dynamic system for bacterial communication? Front Microbiol. 2017;8. https://doi.org/10.3389/fmicb.2017.01454.

82. Campana R, Ciandrini E, Baffone W. Experimental approach for a possible integrated protocol to determine sanitizer activity against both planktonic bacteria and related biofilms. Food Res Int. 2018:111:472-9.

\section{Publisher's Note}

Springer Nature remains neutral with regard to jurisdictional claims in published maps and institutional affiliations.

\section{Ready to submit your research? Choose BMC and benefit from:}

- fast, convenient online submission

- thorough peer review by experienced researchers in your field

- rapid publication on acceptance

- support for research data, including large and complex data types

- gold Open Access which fosters wider collaboration and increased citations

- maximum visibility for your research: over $100 \mathrm{M}$ website views per year

At $\mathrm{BMC}$, research is always in progress.

Learn more biomedcentral.com/submission 